\title{
A Stimulus-Response Framework for Robot Control
}

\author{
MARIO GIANNI, ALCOR Laboratory, DIIAG, Sapienza, University of Rome, Italy \\ GEERT-JAN M. KRUIJFF, Nuance Deutschland GmbH, Aachen, Germany \\ FIORA PIRRI, ALCOR Laboratory, DIIAG, Sapienza, University of Rome, Italy
}

\begin{abstract}
We propose in this article a new approach to robot cognitive control based on a stimulus-response framework that models both a robot's stimuli and the robot's decision to switch tasks in response to or inhibit the stimuli. In an autonomous system, we expect a robot to be able to deal with the whole system of stimuli and to use them to regulate its behavior in real-world applications. The proposed framework contributes to the state of the art of robot planning and high-level control in that it provides a novel perspective on the interaction between robot and environment. Our approach is inspired by Gibson's constructive view of the concept of a stimulus and by the cognitive control paradigm of task switching. We model the robot's response to a stimulus in three stages. We start by defining the stimuli as perceptual functions yielded by the active robot processes and learned via an informed logistic regression. Then we model the stimulus-response relationship by estimating a score matrix that leads to the selection of a single response task for each stimulus, basing the estimation on low-rank matrix factorization. The decision about switching takes into account both an interference cost and a reconfiguration cost. The interference cost weighs the effort of discontinuing the current robot mental state to switch to a new state, whereas the reconfiguration cost weighs the effort of activating the response task. A choice is finally made based on the payoff of switching. Because processes play such a crucial role both in the stimulus model and in the stimulus-response model, and because processes are activated by actions, we address also the process model, which is built on a theory of action. The framework is validated by several experiments that exploit a full implementation on an advanced robotic platform and is compared with two known approaches to replanning. Results demonstrate the practical value of the system in terms of robot autonomy, flexibility, and usability.
\end{abstract}

Categories and Subject Descriptors: I.2.9 [Robotics]: Autonomous Vehicles, Operator Interfaces; I.2.10 [Vision and Scene Understanding]: Perceptual Reasoning, Representations, Data structures, and Transforms; F.4.1 [Mathematical Logic]: Logic and Constraint Programming; I.2.4 [Knowledge Representation Formalisms and Methods]: Predicate Logic, Situation Calculus, Relation System, Representation Languages; I.2.6 [Learning]: Parameter Learning, Induction; I.2.8 [Problem Solving, Control Methods, Search]: Plan Execution, Formation, and Generation

General Terms: Design, Algorithms, Performance, Reliability, Languages

Additional Key Words and Phrases: Task switching, intelligent interaction, stimulus-response model, behavior adaptation

\section{ACM Reference Format:}

Mario Gianni, Geert-Jan M. Kruijff, and Fiora Pirri. 2015. A stimulus-response framework for robot control. ACM Trans. Interact. Intell. Syst. 4, 4, Article 21 (January 2015), 41 pages.

DOI: http://dx.doi.org/10.1145/2677198

This work is supported by the EU-FP7 ICT 247870 NIFTI project.

The reviewing of this article was managed by Lutz Frommberger, Heriberto Cuayahuitl, Nina Dethlefs, Antoine Raux, Matthew Marge, and Hendrik Zender.

Authors' addresses: M. Gianni and F. Pirri, ALCOR LAB, Dipartimento di Ingegneria Informatica, Automatica e Gestionale, "Antonio Ruberti", via Ariosto 25, 00185, Roma; emails: \{Mario.Gianni, Fiora.Pirri\}@ dis.uniroma1.it; G.-T. M. Kruijff, Nuance Communications Deutschland GmbH, Kackertstrasse 10, D-52072 Aachen, Germany; email: gj@nuance.com.

Permission to make digital or hard copies of part or all of this work for personal or classroom use is granted without fee provided that copies are not made or distributed for profit or commercial advantage and that copies bear this notice and the full citation on the first page. Copyrights for components of this work owned by others than ACM must be honored. Abstracting with credit is permitted. To copy otherwise, or republish, to post on servers, or to redistribute to lists, requires prior specific permission and/or a fee. Requested permissions from Permissions@acm.org.

2015 Copyright is held by the owner/author(s). Publication rights licensed to ACM.

ACM 2160-6455/2015/01-ART21 $\$ 15.00$

DOI: http://dx.doi.org/10.1145/2677198 


\section{INTRODUCTION AND MOTIVATIONS}

A robot interacting with people and with the environment is subject to several stimulus events, like any living organism. How these stimuli are to be represented and how a response should be modeled has not yet received the necessary attention, even though robot autonomy, beyond laboratory experiments, relies on an intelligent response to these stimuli.

Everyone knows what a stimulus is in the human and animal sense. The concept of stimulus has been studied the eighteenth century, with the work of Galvani and Volta on frogs, and was further explored in psychology, physiology, and psychophysics. Modern physiology considers a stimulus applied to a receptor and capturing information from both inside and outside the body; these are called proximal and distal stimuli, respectively. Following the pioneering work of Weber [1834], Gustav Fechner introduced psychophysics to model the relation of physical stimuli to the resulting mental facts. Gibson [1960] proposes the following a constructive view of a stimulus:

I think the central question is the following. Is a stimulus that which does activate a sense organ or that which can activate a sense organ? Some writers imply that a stimulus not currently exciting receptors is not a stimulus at all. Others imply that a stimulus need not excite receptors to be called such. [Gibson 1960]

Gibson's implicit question is about the possibility of giving a structure, a definition, to the relationship between objects and stimuli and between stimulus and response:

Perhaps there is an invariant stimulus for the invariant response, after all. Many sorts of higher order variables of energy may exist, only awaiting mathematical description. They will have to be described in appropriate terms, of course, not as simple functions of frequency and amount. We must not confuse a stimulus with the elements used for its analysis. [Gibson 1960]

As a matter of fact, we do not yet have a model of robot stimuli, and most researchers accept that any sensory input from the environment or from the robot's internal states can be considered a stimulus. This simplification is of no help, as the wide literature on human and animal stimuli witnesses. If any sensory input can be considered a stimulus, how it would be possible for a robot to discern between a wall and a victim lying on the ground, between the light from a fire and the light of a lamp, between a scream and repeated noise, and so on? Not all inputs require a response, but a stimulus does.

In this article, we address the problem of providing a representation of what a stimulus is for a robot and how it affects the robot's behavior and its tasks under execution. Consider that whereas for a living organism pain is a stimulus that has a direct effect on its survival awareness, for a robot, a similar stimulus could be the lack of a WiFi connection or battery exhaustion, both indicating a loss of vitality. Therefore, in providing such a representation, we answer the question of how to model the stimulus reflex within the robot's structure and language, and we explore how the robot learns the best stimulus-response according to the context of its current task.

In living organisms, stimulus transduction elicits the stimulus reflex through a receptor. In a robot, this transduction is conveyed by its active processes. Consider the robot motion system, which is made of several components, each of which is driven by processes controlling velocity, steering, obstacle avoidance, terrain adaptation, tracking and so on. These processes collect information from the environment and from the robot's internal states. These processes elaborate the collected information and update it, eventually returning it to the robot's inner states to manage its current activities. In other words, the interaction with environment configures a space of information allocating to each process a quantum of information that is transduced to a stimulus at specific peaks of the information manifold. A stimulus induces a request with the 
call for a response. A response might appeal to robot components and their processes not yet active at the very moment at which the stimulus is triggered.

In this article, we introduce a preliminary framework to model robot processes, their yields, the stimuli occurrences, and the decision underlying the response to the stimuli. The advantage of this approach is that robot control does not need to be designed a priori but instead can be drawn by its interactions with users who teach the robot when a stimulus occurs and what the possible alternatives are for handling the stimuli. These aspects are not dealt with in any other control methods, including planning, whether reactive, proactive, or declarative. Indeed, a robot that has learned a stimulus-response strategy from several humans will most certainly be more usable than a robot that either has no stimuli at all or has no strategy to respond to stimuli. We also take context into account in order to establish a response cost, and we exploit a theory of actions that models how tasks are chosen and how switching to a new task can occur as a result of a stimulus-response.

The ability to selectively respond to several stimuli and to inhibit inappropriate urges by focusing on the task at hand are well-known in humans as shifting and inhibition executive functions, respectively [Miller and Cohen 2007; Aron 2007]. The neuroscience theory of control uses inhibition to explain many cognitive activities, especially to explain how an individual presented with several stimuli responds selectively and is able to resist inappropriate urges [Tipper 2001].

Since the early work of Jersild [1927], several studies in neuroscience and cognitive science have led to a better understanding of many of the variables affecting task switching in the context of cognitive control (we refer the reader to Monsell [2003] and the citations therein). These theories have strongly influenced cognitive robotics architectures since the 1980s, as, for example the ATA schema [Norman and Shallice 1986], the principles of goal-directed behaviors in Newell [1990], and, more recently, the CogX architecture [Hawes et al. 2010; Wyatt et al. 2010]. (For a review on the earliest architectures in the framework of the task switching paradigm, see Rubinstein et al. [2001].)

Only recently has the need to model the stimulus-response, and hence the cognitive control underlying task switching and the cognitive functions of shifting and inhibition, become a hot topic in cognitive robotics. The need to model adaptation, to increase the flexibility in handling complex tasks, has led to modeling task switching for robot navigation, motion planning, and control in large-scale environments [Althaus and Christensen 2003], for path planning [Medina Ayala et al. 2012], for recognizing human intentions in conversation [Nakano et al. 2011], and for motor planning to improve tasks execution [Ganesh and Burdet 2013]. Task switching is also addressed in manipulation [Ajoudani et al. 2013; Ding and Fang 2013] and soft robot control [Tao et al. 2012; Li et al. 2013], for modeling multirobot systems [Sung et al. 2013], for learning task space control through the use of different tools [Jamone et al. 2013], for biped walking in rough terrain [Saglam and Byl 2013], and for multiple-space control [Li and Cheah 2012].

Most of the cited works are quite recent, showing increasing interest in the stimulusresponse framework for coping with adaptivity and ecological behaviors in an interactive environment. Further examples of these studies exist [Capi 2007; Suzuki et al. 2009; Wawerla and Vaughan 2009; Finzi and Pirri 2010; Durkee et al. 2011; D’Ambrosio et al. 2011]. The major problem to be resolved, as noted in Wawerla and Vaughan [2009], is the switching decision. Experts on task switching claim that this decision incurs a switching cost. This cost results from the interplay between the resources needed to reconfigure a mental state and the resources needed to resolve interference with the current state [Monsell 2003]. We model this cost by considering both the reconfiguration of the mental state and the interference with the current state. The reconfiguration 


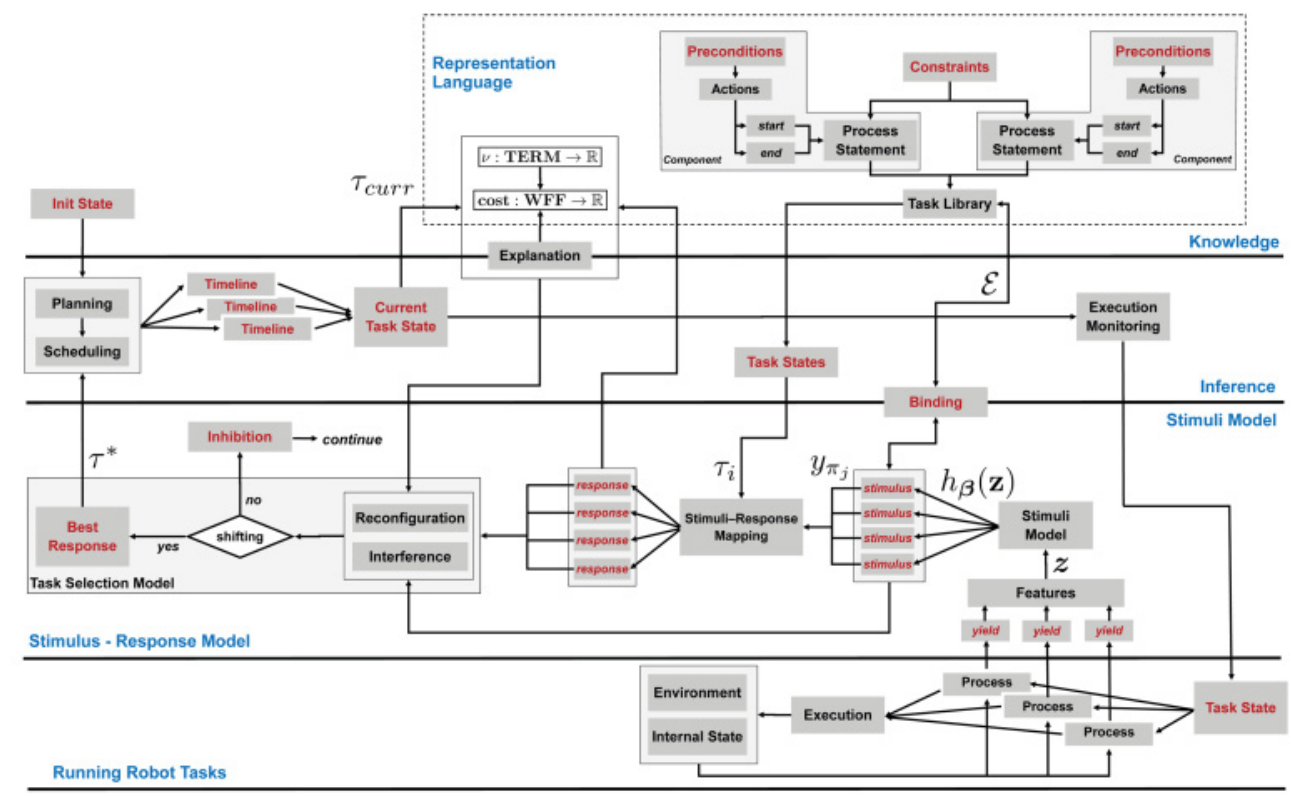

Fig. 1. Work flow of the proposed stimulus-response framework. From bottom to top: the lowest layer indicates a currently active task being executed. The active processes feed the process yields, collecting information from them; the stimulus model selects from the yields those which are stimuli. The stimulusresponse model scores the response tasks. The decision is taken by evaluating the payoff of switching to a task suggested by the stimulus-response matrix. In the middle panel, between knowledge and inference, execution monitoring takes care of the actual task execution and its updating, both affecting the mental states; namely, the decision of whether to consent to a response to the stimulus. In the upper panel, a first-order logical formalism is used to model the robot processes, with action preconditions and effects affecting activation costs and motivating causal constraints. This closes the loop between stimulus activation, reasoning, planning, and decision.

and interference costs are defined in terms of the amount of processes that need to be inferred in order to initiate a new task and their congruency with the current task. The decision is made based on a payoff that is computed by considering the cost of updating the current robot state and the risk of continuing with the task under execution. The stimulus-response model illustrated in these pages indicates a new direction in robot cognitive control, and the tests and experiments presented here prove that this is a promising direction.

The stimulus-response framework introduces new emerging methodologies that build cognitive robot control via learning and perception, and we expect that it will spread to several applications that require cognitive control to resolve the continuous struggle a robot has to face in a real-world environment.

\section{EXECUTIVE SUMMARY}

The flowchart of the proposed robot stimulus-response framework is illustrated in Figure 1. The lower layer represents a robot task under execution. The next level illustrates the stimuli and the response models, described in Sections 3 and 4 . The level enclosed between Inference and Knowledge delineates the work flow linking the decision level to the current execution level. The upper level provides a schema of the system representation in the formal language of Situation Calculus, where the mapping of the stream of information into the domain of reasoning takes place. These levels are described in Sections 5 and 6. 
Table I. Examples of Yields of Processes and Their Features

\begin{tabular}{|l||c|c|c|c|c|}
\hline \hline \multicolumn{3}{|c|}{ Yields } & \multicolumn{2}{c|}{ and Their Features } & Stimulus Description \\
\hline Process & Level & Power Consumption & Location & - & Exhaustion \\
\hline Battery & Strength & Dist. Source & Speed & Traffic & Disconnection \\
\hline WiFi & Resolution & Surface & Density & Smoothness & Lack of data, device error \\
\hline Laser scan & Height & Slope & Intricacy & Depth & 3D data not available, obstacle \\
\hline Mapping & Shape & Dimension & Location & Distance & Identification, Detection \\
\hline Visual perception & Light & Color & Motion & Contour & Saliency \\
\hline Attention & Sound & Id Voice & Utterance & Meaning & Request \\
\hline Interface & Scale & Frequency & Pitch & Who & Mute, Noise \\
\hline Sound & &
\end{tabular}

Each active robot process yields a quantum of information with characteristic features; this quantum of information is called the yield of an active process. The features $\mathbf{z}$ of the yield are used by the stimulus model to learn a function $h_{\beta}(\mathbf{z})$ (Section 3$)$. This function establishes whether or not a stimulus occurred during the specific process execution.

Example 2.1. Consider the robot process $\pi_{W i F i}$ managing the connection between the robot and a remote command base station. We expect that the stimulus occurs when a high signal becomes low or disappears. So the process $\pi_{W i F i}$ yields a quantum of information specified by a set of features $\mathbf{z}_{W i F i}$, such as those indicated in Table I. The robot, with the help of users, learns to discriminate a stimulus from ordinary information via the function $h_{\beta}\left(\mathbf{z}_{W i F i}\right)$, discerning between typical $\mathbf{z}_{W i F i}$ values and odd ones. Therefore, the outcome of the learned function $h_{\beta}$ is a value $y=1$ to fire the stimulus of WiFi disconnection and $y=0$ otherwise.

If the stimulus occurs, the robot has to choose a possible response (e.g., going back to the last position where it was connected), or it can inhibit the stimulus and continue its task even though it is disconnected from the operator. Therefore, there are two issues that need to be modeled:

(1) identify the task that is a possible response to the stimulus;

(2) decide whether to go on with the current task or to switch to the identified response task.

The first item is dealt with by filling a score matrix $\mathbf{A}$ whose values are estimated via factorization, as described in Section 4.

On the other hand, the decision to either shift from the current task to a new task in so responding to the stimulus, or to inhibit the stimulus, is based on the payoff of switching. This is computed considering the risk of continuing the current task, without taking into account the stimulus, and the effort required to fulfill the stimulus. In turn, the effort is computed considering two costs: (1) the cost to reconfigure the current robot state to the new state that switching would lead to and (2) the cost to resolve the interference due to the interruption of the current task. These costs are computed by considering the preconditions and effects of each action involved in both the processes to be interrupted and in the ones to be activated (Section 6). To bind the information yielded by a process to the domain of reasoning, a special functional $\mathcal{E}$ is used, mapping the process terms of the representation language to the corresponding values of both the yields and the stimuli at execution time (Section 5).

Example 2.2. Suppose the robot is executing task $\tau_{10}$ (see Table VII, in Appendix A) that requires the robot to approach a victim in a rescue area. The processes composing this task are listed in Table VII. Assume that the yield of process $\pi_{W i F i}$ (named 
connection management in Table VII) fires a stimulus, namely, the current values of its yields $\mathbf{z}_{W i F i}$ induce $h_{\beta}\left(\mathbf{z}_{W i F i}\right)=1$. Let the stimulus-response matrix $\mathbf{A}$ suggest that the response task is $\tau_{4}$; namely, recovery WiFi signal. To start the task recovery WiFi signal, all the required processes have to be activated with their preconditions and postconditions; therefore, the reasoning system of the robot has to be informed of the stimulus in order to make a decision by computing the payoff of switching. The mapping $\mathcal{E}$, applied to the features, transforms them into values that are terms of the representation language, thus allowing the cost computation to take place.

The article is organized as follows. In the next section, we introduce a representation of the stimuli. In Section 4, we describe the computation of the stimulus-response matrix. In Section 5, we describe the fundamental formalism needed to define concepts such as tasks and processes that are needed to model the transduction between stimuli and processes. In Section 6, we illustrate both how switching costs are computed and how the payoff for switching is obtained. Section 7 evaluates, with some experiments, the performance of the proposed stimulus-response model at managing task switching when incoming information acknowledges new situations to be faced. In particular, results are confronted with replanning at execution time as proposed in the context of CRAM [Beetz et al. 2010] and DTPDDL, implementing POMDP [Talamadupula et al. 2010; Göbelbecker et al. 2011]. The experiments prove the correctness of the robot's behavior in the presence of different stimuli, cover many aspects that have not been adequately treated in the article, and present the whole picture as simply as possible. In the Appendix, we provide some implementation details of the robotic platform and its functionalities; we also use tables to illustrate the implementation of several robot components, their processes, and the stimuli yielded by those processes; finally, we show the stimulus-response matrix developed for a set of stimuli and tasks.

\section{THE STIMULUS MODEL}

In this section, we introduce what we define as a robot stimulus and how we estimate the presence of a stimulus given the information gathered by a process.

Consider the following events applied to a robot, not necessarily in parallel: the battery is getting low, the WiFi has a very weak or absent signal, saliency is detected in a sequence of frames, the memory is surcharged, the communication with the operator is clogged, a request pops up on the interface, and someone is talking at the dialog box. A robot has to deal with hundreds of events like these. These events are stimuli, in the sense that they trigger some alert on the information process transduced by an executive process of an active robot component such as, for the example, the battery, the WiFi, the attention system, the mapping system, the engines, the dialog system, the interface, or the obstacle avoidance system. Three concepts are at the core of stimulus modeling: (1) the information flow caused by an active robot process, (2) the states and events inducing a stimulus, and (3) the rule by which a stimulus is detected to be so:

it is reasonable to assume that stimuli carry information about the terrestrial environment. That is, they specify things about objects, places, events, animals, people, and the actions of people. The rules by which they do so are to be determined, but there is at least enough evidence to warrant discarding the opposite assumption under which we have been operating for centuries that stimuli are necessarily and intrinsically meaningless. [Gibson 1960]

Consider a robot process $\pi$ being executed among a number of others under execution. This process $\pi$ has a domain of interest defined by the values it controls; some example are given in Table I. These values are the realizations of a stochastic variable $\mathbf{X}: \Omega^{d} \mapsto$ $\mathbb{R}^{d}$, and we identify them as the yield of the active process $\pi$. At each instant time $t$, 
if the process is active, then the stochastic variable is realized and the values it takes are known; either they induce a stimulus for the process $\pi$ or they do not.

More precisely, the yield of a process $\pi$ forms an observation vector $\mathbf{z}=\left(z_{1}, \ldots, z_{d}\right)^{\top}$, $\mathbf{z} \in \mathbb{R}^{d}$ and whether these values induce a stimulus or not is the objective of a function $h_{\beta}: \mathbb{R}^{d} \times \boldsymbol{\beta} \mapsto[0,1]$ to be learned with parameters $\boldsymbol{\beta}=\left(\beta_{0}, \beta_{1}, \ldots, \beta_{d}\right)^{\top}$ and arguments being the yield of the process.

A trial is the set of observations gathered in a discrete time interval $T$; if $n$ trials are performed by $n$ operators, we obtain a training set $\mathcal{T}$ of size $(n T \times(d+1))$. The training set is of the form $\mathcal{T}=\left(\left\langle\mathbf{z}_{1}, y_{1}\right\rangle, \ldots,\left\langle\mathbf{z}_{n T}, y_{n T}\right\rangle\right)^{\top}$, with $\mathbf{z}_{i} \in \mathbb{R}^{d}, y_{i} \in\{0,1\}$.

The logistic regression model can be used to estimate the outcome $y$. We recall that the logistic regression model computes the probability of the outcome $y$ as:

$$
h_{\beta}(\mathbf{z})=\mathcal{B}(g(a)),
$$

where $\mathcal{B}(x)$ is the Bernoulli density $\mu^{x}(1-\mu)^{(1-x)}$ with $\mu \in[0,1] ; a$ is the activation, namely, $a=\boldsymbol{\beta}^{\top}\left(1, \mathbf{z}^{\top}\right)^{\top}$; and $g$ is the sigmoid function:

$$
g(a)=\frac{1}{1+\exp (-a)}
$$

The goal is to estimate the parameters $\beta$ of the function $h_{\beta}$.

It is important to note that for this model to predict accurately the occurrence of a stimulus with the outcome value $y$, given the features $\mathbf{z}$ of the specific process yields, it is required that the outliers introduced during data gathering are kept under control before the estimation of the parameters $\beta$ takes place. In fact, in addition to the difficulty of obtaining comparable trials, the training set $\mathcal{T}$ is extremely noisy due to operators mistakes made while a trial is running.

Since the noise is mainly introduced by the operator in the difficult task of deciding whether an observation is or not a stimulus, we have devised a method of rejecting most of the outliers that severely affect parameter estimation. Indeed, we exploit some prior knowledge about stimuli/nonstimuli for each process, and we use this to define a function that is consistent with the typical behavior of the process and that rejects wrong data in the training set. The proposed function maps to a rejection region all those outcomes that are classified as stimuli but whose range of yields should in principle assign them to a nonstimuli classification since stimuli are extraordinary events. The function defining the rejection region is:

$$
\gamma(\mathbf{z}, y)= \begin{cases}1 & \text { iff } y=1 \text { and } \mathbf{z} \notin \text { RejRegion } \\ 1 & \text { iff } y=0 \\ 0 & \text { otherwise. }\end{cases}
$$

Here, RejRegion is the rejection region that still need to be defined. This is done according to the following steps:

(1) Fit nonparametric kernel density to the data $\left(\mathbf{z}_{1}, \ldots, \mathbf{z}_{n T}\right)$ using a Gaussian kernel.

(2) Find the modes of the density. The modes indicate where the data are more dense; in other words, they indicate the values identifying the most typical behavior of the process yield.

(3) Select the data falling in the regions around the modes $\left\{\hat{\mathbf{z}}_{q}\right\}, q=1, \ldots, k_{M}$, with $k_{M}$ being the number of modes, and lock these data into an hyperellipsoid whose dimension is estimated according to the Hotelling test, assuming that these data come from a Gaussian distribution.

(4) The confidence region for the mean of the selected data is the required rejection region RejRegion. 
For the first step, we consider a smoothing kernel over the process yield on a $d$ dimensional grid. Let $\mathbf{x}=\left(x_{1}, \ldots, x_{d}\right) \in \mathbb{R}^{d}$ be a point in the grid, and let $\mathbf{z}_{k} \in \mathbb{R}^{d}$ be an observed yield, $1 \leq k \leq n T=N$; let $v=\left[\left(\frac{\mathbf{x}-\mathbf{z}_{k}}{h^{d}}\right)^{\top}\left(\frac{\mathbf{x}-\mathbf{z}_{k}}{h^{d}}\right)\right]^{1 / 2}$, with $h>0$ the kernel window. We consider a radial symmetric Gaussian kernel $K_{h d}(v)=\frac{1}{\sqrt{2 \pi}} \exp \left(-\frac{1}{2} v^{2}\right)$. A nonparametric estimate for the observations that are close to point $\mathbf{x}$ is:

$$
f_{h d}\left(x_{1}, \ldots, x_{n}\right)=\frac{1}{N} \sum_{k=1}^{N} c K_{h d}\left(v_{k}\right)
$$

Here, $c$ is the normalization term that, by the fact that $\int_{-\infty}^{\infty} K(v) d v=1$, is $\left(h^{d^{2}}(2 \pi)^{(d-1) / 2}\right)^{-1}$. The gradient of the density is

$$
\nabla f_{h d}\left(x_{1}, \ldots x_{d}\right)=\left\{\frac{c}{N} \sum_{k=1}^{N}-\frac{1}{h^{2 d}} K_{h d}\left(v_{k}\right)\left(x_{j}-z_{k j}\right)\right\}_{j=1, \ldots, d}
$$

Then, by some simple algebraic transformation and hiding in $c$ the constant term we obtain:

$$
\nabla f_{h d}\left(x_{1}, \ldots, x_{d}\right)=\left\{f_{h d}\left(x_{1}, \ldots, x_{d}\right)\left(\frac{\sum_{k=1}^{N} K_{h d}\left(v_{k}\right) z_{k j}}{\sum_{k=1}^{N} K_{h d}\left(v_{k}\right)}-x_{j}\right)\right\}_{j=1, \ldots, d .}
$$

Local maxima of $f_{h d}$ are computed by gradient ascent; these are the modes $\widehat{\mathbf{z}}$ of the function $f_{h d}$. Let us consider these modes $\left\{\widehat{\mathbf{z}}_{q}\right\}, q=1, \ldots, k_{M}$, with $k_{M}$ the number of modes. Assume these data come from a Gaussian distribution with means $\mu_{q}$ and covariances $\Sigma_{q}$, and let $\overline{\mathbf{z}}_{q}$ and $\mathbf{S}_{q}$ be, respectively, the sample mean and sample covariance of the $q$-th region under the $q$-th mode. The confidence region at level $(1-\alpha)$ for the data mean $\mu_{q}$ is:

$$
C R_{q}=\left\{\mu_{q} \in \mathbb{R}^{d} \mid\left(\mu_{q}-\overline{\mathbf{z}}_{q}\right)^{\top} \mathbf{S}_{q}^{-1}\left(\mu_{q}-\overline{\mathbf{z}}_{q}\right) \leq \frac{d}{N-d} F_{(1-\alpha) ; d, N-d}\right\}, q=1, \ldots, k_{M} .
$$

Here, $F_{(1-\alpha) ; d, N-d}$ is the $1-\alpha$ quantile of the $F$-distribution with $d$ and $N-d$ degrees of freedom. That is, we are confident at a level $(1-\alpha)$ that $C R_{q}$ contains $\mu_{q}$; namely, $p\left(\mu_{q} \in C R_{q}\right)=(1-\alpha)$. Now, $\left(\mu_{q}-\overline{\mathbf{z}}_{q}\right)^{\top} \mathbf{S}_{q}^{-1}\left(\mu_{q}-\overline{\mathbf{z}}_{q}\right), q=1, \ldots, k_{M}$ are hyperellipsoids with center $\mu_{q}$ and semi-axes of length $\sqrt{D_{q}^{2} \lambda_{s q}}$, where $\lambda_{s q}$ is the $s$-th eigenvalue of $S_{q}^{-1}$. Since $D_{q}^{2}=\frac{d}{N-d} F_{(1-\alpha) ; d, N-d}$ is the maximal ellipsoid surface, enclosing the $q$-th confidence region, it follows that these regions correspond to the most typical behavior of the yield of the process $\pi$ under consideration. Hence, these are rejection regions for stimuli-regions where it is most unlikely that a stimulus can occur. Therefore, we are finally arrived at the definition of rejection region RejRegion:

$$
\text { RejRegion }=\left\{\mathbf{z} \in \mathbb{R}^{d} \mid\left(\overline{\mathbf{z}}_{q}-\mathbf{z}\right)^{\top} \mathbf{S}_{q}^{-1}\left(\overline{\mathbf{z}}_{q}-\mathbf{z}\right) \leq D_{q}^{2}, q=1, \ldots k_{M}\right\} .
$$

As noted earlier, $k_{M}$ is the number of modes of the nonparametric density. Therefore, according to Equation (3), the set of samples for which $\gamma\left(\mathbf{z}_{k}, y_{k}\right)>0, k=1, \ldots, N$ (recall that $N=n T$ ) is the optimal one for classification.

Now, given a complete set of good samples $\mathcal{T}^{\star}=\left(\left\langle\mathbf{z}_{1}, y_{1}\right\rangle, \ldots,\left\langle\mathbf{z}_{N}, y_{N}\right\rangle\right)$, the model for stimulus prediction is provided by logistic regression. Namely, the logistic regression coefficients $\beta$ are obtained from the good trial set $\mathcal{T}^{\star}$ for the yield of process $\pi$ by 
Table II. Components, Processes, and Stimuli

\begin{tabular}{|l|c|c|}
\hline Component Description & Process Description & Stimulus Description \\
\hline \hline Battery & Power Level Monitoring & Battery Exhaustion \\
\hline WiFi & Connection Management & WiFi Disconnection \\
\hline Robot Engine & Temperature Monitoring & Temperature Level \\
\hline Visual Perception & Detecting Object/Person & Detected Object/Person \\
\hline Memory & Data Writing & Write Access to Memory Full \\
\hline Dialogue & Human to Robot Exchanging msg & Human to Robot Message \\
\hline
\end{tabular}

minimizing the following cost function:

$$
L(\boldsymbol{\beta})=-\frac{1}{n}\left(\sum_{i=1}^{N} y_{i} \log h_{\beta}\left(\mathbf{z}_{i}\right)+\left(1-y_{i}\right) \log \left(1-h_{\beta}\left(\mathbf{z}_{i}\right)\right)\right)+\frac{\xi}{2 n} \sum_{j=1}^{d} \beta_{j}^{2} .
$$

Here, $\xi$ is the regularization parameter and $\sum_{j=1}^{d} \beta_{j}^{2}$ the regularization term; these are used just in case the features induce a high-dimensional space, which in general is kept $\leq 5$ to manage the nonparametric density. The Newton-Raphson algorithm used to minimize Equation (9) requires an update step expressed as a least square step, namely, as an iteratively reweighted least square. Then, given a new observation $\mathbf{z}^{\star}, h_{\beta}\left(\mathbf{z}^{\star}\right)$ is the hypothesis returning the binary response within the variable $y$. More precisely:

$$
y=\left\{\begin{array}{l}
1 \text { if } h_{\beta}\left(\mathbf{z}^{\star}\right) \geq 0.5 \\
0 \text { if } h_{\beta}\left(\mathbf{z}^{\star}\right)<0.5
\end{array}\right.
$$

We have thus provided a model to represent the stimuli and for learning when such stimuli occur. We have also introduced the principle that a large region of nonstimuli for each process yield can be identified around the most typical values of the yield to simplify noisy data collection. Note that this concept of typical values for the process yield grants the possibility of estimating $h_{\beta}$ with quite few trials.

\section{THE STIMULUS-RESPONSE MATRIX}

Given a process $\pi$, we have introduced the function $h_{\beta}$ that takes as input the yield of a process and determines whether a stimulus is triggered or not, returning a value 1 if this is the case and a value 0 otherwise. If a stimulus occurs, it is necessary to score all possible response tasks before any decision can be taken.

In this section, we show how to score the relation stimulus response, which amounts to scoring the task that would eventually be chosen to respond to the triggered stimulus. Here, a task is a set of processes; see Table II for an excerpt of the structures linking robot components, processes, and stimuli, and the more detailed Tables VI, VII, and VIII in Appendix A. Therefore, while a task is being executed, a stimulus can be triggered from any of the active processes of the task. To address each case, given a limited number of tasks for a robot, we introduce a stimulus-responses matrix $\mathbf{A}$ that scores each pair: The score matrix $\mathbf{A}$ is formed by two classes of entities, which we refer to as Tasks (rows) and Stimuli (columns), respectively (see Table VII and Table VIII in Appendix A). Note that a task is built out of many processes, yet each process can yield a single stimulus; namely, there is a bijection between each process and the stimulus it yields.

Matrix $\mathbf{A}$ is initially built using a number of experiments and further completed by estimating the unknown values for the stimulus-response pairs with the regularized low-rank matrix factorization method [Zhang et al. 2012; Chen 2008; Buchanan and Fitzgibbon 2005; Canny 2002]. 
The experiments for filling $\mathbf{A}$ are short-term missions performed by operators who are well informed about the task library, the processes, and the stimuli that the robot can handle. Each operator is assigned a task from the task library (see Table VII in Appendix A, as described in Section 7). During the trial, a stimulus is triggered for an active process $\pi_{j}$. Upon the occurrence of the stimulus, the operator selects the task $\tau_{i}$ that he or she considers the most appropriate to respond to the presented stimulus from among those reported in Table VII (Appendix A) using a graphical user interface (see Figure 11 in Appendix A). Note that, during the experiment, the operator teleoperates the robot. The experiment terminates when the operator signifies his or her task choice. Data from each trial, reporting the occurrence of the pairs $\left\langle\tau_{i}, y_{\pi_{j}}\right\rangle$, are collected, and the corresponding entries $a_{i j}$ of matrix $\mathbf{A}$ (see Table IX and Table X) are filled. When operators did not select the corresponding stimulus-task pair, a dash sign is added.

The problem now is to complete the matrix by finding the missed values. These are recovered by factorizing matrix $\mathbf{A}$ into the product of two smaller matrices, $\mathbf{P} \in \mathbb{R}^{m \times d}$ and $\mathbf{Q} \in \mathbb{R}^{n \times d}$, of rank $d^{1}$. Given that $\mathbf{A}$ has dimension $(n \times m)$-namely, $n$ tasks and $m$ process yields (here, $n=13$ and $m=42$ ) —and rank $d$, in order to complete $\mathbf{A}$, it is necessary to find two factors explaining $\mathbf{A}$ : an $(n \times d)$ matrix $\mathbf{Q}$ and an $(m \times d)$ matrix $\mathbf{P}$, such that $\mathbf{A}=\mathbf{Q} \mathbf{P}^{\top}$. Thus, it is possible to minimize the error norm on the known values.

Let $\mathbf{W}$ be an indicator matrix of the same size as $\mathbf{A}$ in which 0's correspond to missing elements of $\mathbf{A}$ and 1's correspond to observed data; then, the problem can be reformulated as:

$$
\epsilon(\boldsymbol{Q}, P)=\left\|W \odot\left(\mathbf{A}-\mathbf{Q} \mathbf{P}^{\top}\right)\right\|_{F}^{2}+\lambda_{1}\|\mathbf{Q}\|_{F}^{2}+\lambda_{2}\|\mathbf{P}\|_{F}^{2} .
$$

Here, $\odot$ is the Hadamard component-wise matrix product, $\|\cdot\|$ is the Frobenius norm, and $\lambda_{1}$ and $\lambda_{2}$ are regularizing constants. Several approaches have been proposed to minimize this error, considering that if one of $\mathbf{Q}$ or $\mathbf{P}$ are known, there is a closed-form solution for the other that minimizes Equation (11) (see, e.g., the dumped Newton algorithm introduced by Buchanan and Fitzgibbon [2005] and the Wieberg algorithm of Takayuki Okatani and Deguchi [2006]). Here, we have chosen a version of the alternating-least-squares with weighted- $\lambda$-regularization [Zhou et al. 2008], in which the steps to obtain $\mathbf{Q}$ and $\mathbf{P}$ are summarized as follows. Initialize $\mathbf{Q}$ and $\mathbf{P}$ to small random values and let $w_{i j}$ be the $(i, j)$ element of the indicator matrix $\mathbf{W}$; then, the schema (11) can be rewritten as follows:

$$
\left.\epsilon\left(\mathbf{q}_{1}, \ldots, \mathbf{q}_{n}, \boldsymbol{\rho}_{1}, \ldots, \boldsymbol{\rho}_{m}\right)\right)=\frac{1}{2} \sum_{(i, j)} w_{i j}\left(\boldsymbol{\rho}_{j}^{T} \mathbf{q}_{i}-a_{i j}\right)^{2}+\frac{b_{1}}{2} \sum_{i=1}^{n} \sum_{k=1}^{d} q_{i k}^{2}+\frac{b_{2}}{2} \sum_{j=1}^{m} \sum_{k=1}^{d} \rho_{j k}^{2} .
$$

Here, the first term of Equation (12) corresponds to the square error term, and the last terms are the regularization terms of $\mathbf{q}_{i}$ and $\rho_{j}$, respectively. The terms $b_{1}$ and $b_{2}$ are the regularization parameters. Local optima of the objective function in Equation (12) are found via Newton-Raphson gradient descent. The update rules, for every $i=1, \ldots, n$ and for every $j=1, \ldots, m$ are given by the following equations:

$$
\begin{aligned}
q_{i k} & :=q_{i k}-\zeta_{1}\left(\sum_{j} w_{i j}\left(\rho_{j}^{T} \mathbf{q}_{i}-a_{i j}\right) \rho_{j k}+b_{1} q_{i k}\right) \\
\rho_{j k} & :=\rho_{j k}-\zeta_{2}\left(\sum_{i} w_{i j}\left(\boldsymbol{\rho}_{j}^{T} \mathbf{q}_{i}-a_{i j}\right) q_{i k}+b_{2} \rho_{j k}\right)
\end{aligned} .
$$

\footnotetext{
${ }^{1}$ Here, the letter $d$ is used for the matrix rank, while in the previous section it was used to define the size of observations.
} 


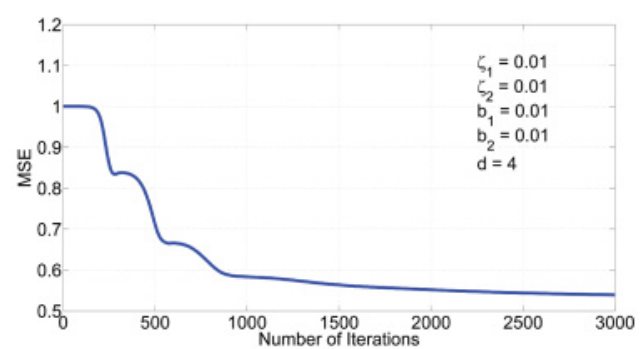

(a)

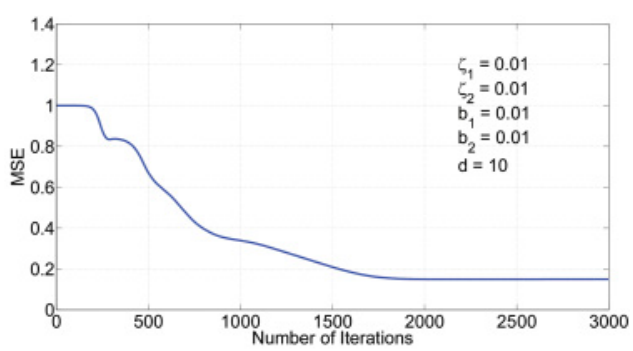

(b)

Fig. 2. Estimation of accuracy of the parameters $\zeta$ and $b$ obtained in Equation (13) given a model with (a) rank of $\mathbf{A}=4$ and (b) rank of $\mathbf{A}=10$.

Once both $\mathbf{q}_{i}$ and $\rho_{j}$ have been estimated, then the score matrix $\mathbf{A}$ can be completed by estimating the missing values of the rates $\widehat{a}_{i j}$ as

$$
\widehat{a}_{i j}=\boldsymbol{\rho}_{j}^{\top} \mathbf{q}_{i} \quad \forall w_{i j}=0 .
$$

The empirical prediction quality is typically low (see Figure 2(a)). Note that, as expected, the Mean Squared Error (MSE) considerably decreases with a higher rank $d$ of the matrix $\mathbf{A}$ and, consequently, the empirical prediction quality gets higher (see Figure 2(b)). Figures 2 shows the accuracy of the prediction when varying the rank of matrix A.

An example of the score matrix $\mathbf{A}$ filled by the trials values for the implemented task library with their processes and stimuli is illustrated in Tables IX and X, in Appendix A. The completed stimulus-response matrix, with the whole score set completed, is shown in Tables XI and XII in the Appendix A.

\section{STATES, PROCESSES, AND TASKS}

The state of the robot is the set of processes that are active at a specific interval time $\Delta t=\left[t^{-}, t^{+}\right]$, and it coincides with the current task in execution (see the tasks given in Table VII in Appendix A). The decision to switch or not to a response task, chosen according to the score matrix $\mathbf{A}$ introduced in the previous section, is a matter of how difficult it is to switch, how many processes need to be interrupted, and how many new processes need to be activated.

To understand the complications intervening in this decision, we briefly introduce the representation of processes in the framework of their control, execution, and monitoring, together with the reasoning mechanism required to infer the facts that need to be appraised before eventually switching to a new task. Namely, switching requires us to infer an appropriate explanation for enabling all the preconditions for activating the response task. In this section, we introduce the following concepts to address this problem:

(1) The representation of actions and processes.

(2) The mechanism generating processes to execute a chosen task.

(3) The constraints between processes working in parallel.

(4) The representation of the robot's current task state.

Tasks and processes are modeled in the action theory $\mathcal{A}$ based on the formalism of Situation Calculus. Planning, the decision to switch, and execution monitoring are all expressed in Situation Calculus. In this formalism, the semantic domain is partitioned in sorts $(A, S, T, U, W)$ where $A$ stands for actions, $S$ for situations, $T$ for time, $U$ for the set of boolean values $\{0,1\}$, and $W$ for every other object. Given the sorted domain, the representation language $\mathcal{L}$ (via its signature $\Sigma_{\mathcal{L}}$ ) is made of predicates and terms 
(variables and functions of arity $n \geq 0$ ) and ruled by the first-order predicate calculus, with the exception of an inductive axiom. We refer the reader to Pirri and Reiter [1999] for a full description of the formalism. The representation language is used to provide the robot with a reasoning and knowledge structure about actions by specifying their preconditions and effects, and about both the processes and the constraints linking their parallel execution.

A process is a predicate $\pi(\mathbf{x}, u, t, d o(a, s))$ taking as arguments a single term $u$ of sort boolean for the stimulus, a term $t$ of sort time, a single term $d o(a, s)$ of sort situation, and terms $\mathbf{x}=\left(x_{1}, \ldots, x_{n}\right), n \geq 0$ of any other sort. The special function do of sort situation is an action sequence builder that defines a situation as a list of actions. More precisely, $d o$ is a function taking as arguments a term of sort action and a term of sort situation and returning a term of sort situation $d o: A \times S \mapsto S$, which is a list of actions. Actions are functions, and when the term of sort situation explicitly mentions the sequence of actions it builds, we denote the term with $\sigma$. Through its arguments, a process carries the information about the history of all processes of a component via all its start and end actions, activated up to the time $t$. For example, consider one of the processes given in Table VI, Appendix A: The connection management of the WiFi component is represented in language $\mathcal{L}$ by the following transition statement:

$$
\begin{aligned}
\pi_{W i F i}(\mathbf{x}, u, t, d o(a, s)) & \equiv\left[a=\operatorname{start}_{W i F i}(\mathbf{x}, t) \wedge u=0\right] \vee\left(\exists t^{\prime} . t^{\prime} \leq t \wedge \pi_{W i F i}\left(\mathbf{x}, u, t^{\prime}, s\right)\right. \\
& \left.\wedge\left(\forall t^{\prime \prime} \forall u .\left(t^{\prime \prime}>t^{\prime} \wedge t^{\prime \prime} \leq t\right) \rightarrow \mathcal{E}\left(\mathbf{x}, t^{\prime \prime}\right)=u \rightarrow a \neq \operatorname{end}_{W i F i}\left(\mathbf{x}, t^{\prime \prime}\right)\right)\right) .
\end{aligned}
$$

Here, start $t_{W i F i}$ and $e n d_{W i F i}$ are actions, and $t, t^{\prime}, t^{\prime \prime}$ are variables of sort time. The function $\mathcal{E}$ maps terms of the representation language $\mathcal{L}$ to the values identified by the stimulus model (Section 3). This function takes as input the realization of the yields of the process when the process is active, and it returns the value $u=1$ if the signal elicits a stimulus and $u=0$ otherwise. This is called a transition axiom, and it specifies that the robot process managing WiFi connection is active if either the action start WiFi $_{\text {i }}$ is executed at time $t$, or the process was already active at some previous time $t^{\prime}$, and, at any time between $t^{\prime}$ and $t$ (the current time), it was not ended by a disconnection notified by a stimulus nor it was ended by the action end $d_{W i F i}$ independently of the stimulus value. The transduction of a stimulus realization to a term of the language $\mathcal{L}$ requires the existence of a set of transformations binding terms and domains at execution time. This is better detailed by the following remark.

Remark 5.1 (Transduction between stimuli and processes). A process yield and its stimulus value are both linked to the signature $\Sigma_{\mathcal{L}}$ of the action language $\mathcal{L}$ by the mapping $\mathcal{E}$. The domain of $\mathcal{L}$ and of the processes' yields are linked by the interpretation $I$ of $\mathcal{L}$.

To prove this, we first introduce some auxiliary mappings. Let $L$ be a set of labels, and let $T$ be time. We introduce the function $\mathcal{E}^{+}: \mathbb{R}^{n} \times L \rightarrow \Sigma_{\mathcal{L}}$, the function $\mathcal{E}^{\star}$ : $[0,1] \times T \rightarrow \Sigma_{\mathcal{L}}$, and finally the mapping re: $\mathbb{R}^{n} \times T \mapsto \mathbb{R}^{n}$ and the mapping $\mathcal{E}$ : $\Sigma_{\mathcal{L}} \times T \mapsto \Sigma_{\mathcal{L}}$. To these functions, we add the interpretation function $I$ mapping terms of the language into the interpretation domain, and we assume that equality is interpreted by itself. We have to show that, via $\mathcal{E}^{\star}, r e$, and $\mathcal{E}^{+}, \mathcal{E}$ links the feature terms with terms of $\mathcal{L}$ and that the interpretation links, via the mappings, the sample domain where the yields take values with the domain of interpretation for $\mathcal{L}$. Let us assign to each defined process $\pi \in \mathcal{L}$ a label, and let $\mathbf{X}: \Omega^{n} \mapsto \mathbb{R}^{n}$ be a multivariate random variable whose realizations are the observed values $\mathbf{z}$ of the yield of $\pi$ at time $t$. The function re serves to specify the realization with respect to the process yields and time, namely, $\operatorname{re}(\mathbf{X}, t)=\mathbf{z} \in \mathbb{R}^{n}$. Now $\mathcal{E}^{+}$maps real numbers, labeled by the specific process of which they are the yield, into terms of the language such as 
$\mathcal{E}^{+}\left(\left[z_{3}, z_{4}\right], L_{W i F i}\right)=$ (signal_strength,distance_from_source $)$ and so on. Similarly, $\mathcal{E}^{\star}$ takes the outcome of the learning function $h_{\beta}(\mathbf{z})$ (see Section 3) and maps it to a term of the language. These terms have a semantics in the domain $D^{n}$ of $\mathcal{L}$, so that real values labeled by a process name need to be paired with the interpretation of the terms of the specific process. So, for example, the interpretation of the term signal_strength $\in \Sigma_{\mathcal{L}}$, taken as argument by the process $\pi$, has to be linked to the corresponding element of the sample domain $\Omega^{n}$. Therefore, when the process is active, then $t$ is instantiated, the transduction closes the loop between the different terms, and binding occurs on the appropriate instances in the respective domains according to the sorts requirements:

$$
\begin{aligned}
& r e(\mathbf{X}, t)=\mathbf{z} \in \mathbb{R}^{n} \\
& \mathcal{E}^{+}(\mathbf{z}, L)=\mathbf{x} \in \Sigma_{\mathcal{L}} \\
& \mathcal{E}^{\star}\left(h_{\beta}(\mathbf{z}), t\right)=u \in \Sigma_{\mathcal{L}} \\
& u^{I} \in\{0,1\} \subset D \\
& \mathcal{E}\left(\mathcal{E}^{+}(\operatorname{re}(\mathbf{X}, t), L), t\right)=\mathcal{E}(\mathbf{x}, t)=\mathcal{E}^{\star}\left(h_{\beta}(\mathbf{z}), t\right)=u \in \Sigma_{\mathcal{L}} \\
& \mathcal{E}^{I}\left(\mathcal{E}^{+}(r e(\mathbf{X}, t), L), t\right)^{I}=\mathcal{E}^{\star}, I\left(h_{\beta}(\mathbf{z}), t\right)^{I}=u^{I} \in\{0,1\} \subset D .
\end{aligned}
$$

This shows that the mappings $\mathcal{E}^{\star}, \mathcal{E}^{+}$, re, and $\mathcal{E}$, together with the interpretation $I$ of the language, are sufficient to define the transduction between stimuli and processes, both linking the terms of the language and mapping the outcome into $\{0,1\} \subset D$.

A process $\pi$ is active between a start $t_{\pi}$ and an end $_{\pi}$ action. To activate a start or end action, a special predicate Poss tells the robot whether the action can be done given the current situation $s$ and time $t$, for example:

$$
\begin{aligned}
& \text { Poss }\left(\text { end }_{W i F i}(\mathbf{x}, t), u, s\right) \equiv \\
& \quad(\text { at }(\text { home }, t, s) \wedge \neg \text { ask }(\text { operator }, t, s) \vee u=1) \wedge \pi_{W i F i}(\mathbf{x}, u, t, s)
\end{aligned}
$$

tells the robot that the WiFi process can be ended if it is at home and if, for example, there are no operator requests running or a disconnection signal is detected. This statement is called a precondition axiom. Note that a state is defined by all the predicates (processes and facts) that are verified at the specific situation and time in the intended model. Thus, if $\mathcal{M}$ is the model of the language satisfying the whole theory, we might say that $\mathcal{M}=\bigwedge \varphi(t, \sigma)$ to indicate that the finite set of sentences $\bigwedge \varphi$ holds at time $t$ in situation $\sigma$ in the model, and so specify a finite state of the system. On the other hand, we can also write $\mathcal{A} \models \exists \mathbf{x}, u, t . \pi_{C}(\mathbf{x}, u, t, \sigma)$ to indicate that all the models (actually the intended model of the axiomatization) of the action theory $\mathcal{A}$ are also models of the process $\pi_{C}$ at some time $t$ and situation $\sigma$, thus indicating that the situation $\sigma=d o\left(A_{n}(\mathbf{x}), d o\left(A_{n-1}(\mathbf{x}), \ldots, d o\left(A_{1}(\mathbf{x}), S_{0}\right)\right) \ldots\right)$ returns a sequence of actions that governs the processes that can be activated in $S_{0}$ to perform some task involving the component $C$.

Robot components are both hardware devices and software modules such as engines, dialog, vision, mapping, planning, path planning, and so on, all handling several processes and needing to be regulated not only by transition and precondition axioms, but also by both temporal and spatial relations. To handle relations between processes, a parallelization of situations into several timelines has been introduced [Finzi and Pirri 2010], and each timeline assesses the evolution of all the processes of a component. A timeline is a special situation taking into account only the start and end actions of the processes of a component. Parallel situations are handled by a set of situations called bags of timelines. Time constraints are based on the definition of the greatest start time and least end time, thus bounding a process activity within a start and end time, up to a fixed time $t$. The greatest start time is defined as:

$$
\text { greatestStart }_{\pi}(\mathbf{x}, a, t) \stackrel{\text { def }}{=} \exists t^{\prime} \cdot a=\operatorname{start}_{\pi}\left(\mathbf{x}, t^{\prime}\right) \wedge \neg \exists t^{\prime \prime}, a^{\prime \prime} . t^{\prime} \leq t^{\prime \prime} \leq t \wedge a^{\prime \prime}=\operatorname{end}_{\pi}\left(\mathbf{x}, t^{\prime \prime}\right) .
$$


An analogous definition is given for leastEnd, the least end time for an action, thus characterizing the $\Delta t$ inside which a process is certainly active or certainly not active. Given this definition, a time relation such as during is defined as follows:

$$
\begin{aligned}
\left\{\pi_{i}(\mathbf{x}, u, t, \operatorname{do}(a, \sigma))\right. \text { during } & \left.\pi_{j}\left(\mathbf{x}^{\prime}, u^{\prime}, t^{\prime}, \operatorname{do}\left(a^{\prime}, \sigma^{\prime}\right)\right)\right\}\left[\$, \Phi^{\prime}\right] \stackrel{\text { def }}{=} \\
& \sigma \in \Phi \wedge \sigma^{\prime} \in \Phi^{\prime} \wedge \pi_{i}(\mathbf{x}, u, t, \operatorname{do}(a, \sigma)) \wedge \pi_{j}\left(\mathbf{x}^{\prime}, u^{\prime}, t^{\prime}, \operatorname{do}\left(a^{\prime}, \sigma^{\prime}\right)\right) \wedge \\
& {\left[\operatorname{greatestStart}_{\pi_{i}}(\mathbf{x}, a, t) \geq \operatorname{greatest} \operatorname{Start}_{\pi_{j}}\left(\mathbf{x}^{\prime}, a^{\prime}, t^{\prime}\right)\right] \wedge } \\
& {\left[\text { leastEnd }_{\pi_{i}}(\mathbf{x}, a, t) \leq \text { leastEnd } \pi_{j}\left(\mathbf{x}^{\prime}, a^{\prime}, t^{\prime}\right)\right] . }
\end{aligned}
$$

Here, $s$ and $\Phi^{\prime}$ are bags of timelines; specifically, timelines of different robot components. Note that in the formulas specifying constraints (as in Equation (19)), the variables have a lambda binding, which means that the variables are bound by the quantifiers inside the sentence in which they are embedded because the time constraints are macro sentences of the language. Temporal constraints are typically those defined in Allen [1983], among which we consider the set \{during, before, meet, start, finishes, overlaps, equal\}.

For example, the Equation (20) says that to start the process of approaching a certain location, the path planner process needs to be active, which in turns requires the mapping to be active:

$$
\begin{aligned}
& {\left[\pi_{\text {path-planning }}(\mathbf{x}, u, t, \sigma) \text { during } \pi_{\text {mapping }}\left(\mathbf{x}^{\prime}, u^{\prime}, t^{\prime}, \sigma^{\prime}\right)\right]} \\
& \quad \text { before } \pi_{\text {approach }}\left(\mathbf{x}^{\prime \prime}, u^{\prime \prime}, t^{\prime \prime}, \sigma^{\prime \prime}\right)\left[\mathbb{s}, \mathbb{s}^{\prime}, \mathbb{s}^{\prime \prime}\right]
\end{aligned}
$$

A constraint formula is indicated by $\pi_{i} \mathbf{o p} \pi_{j}\left[\Phi_{i}, \Phi_{j}\right]$, and a set of constraint formulas is indicated by $\mathcal{C}$. Note that constraints are special formulas because only the definiens (namely, the left hand side of the definitions) are expressed in the language $\mathcal{L}$.

Different approaches for defining constraints in a first-order language have been proposed [Baar et al. 2001; Block et al. 2006; Morris et al. 2001; McDermott 2003; Fox and Long 2003; Carbone et al. 2008; Wittocx et al. 2010]. A definition for each time constraint between processes, according to linear interval-based time [Allen 1983], is provided in Finzi and Pirri [2010].

Similar to temporal constraints, spatial constraints are defined following the topological relations introduced in Randell et al. [1992]. Spatial and time constraints (see Wolter and Zakharyaschev [2000] and Finger and Gabbay [1996]) are integrated in the framework.

Now we must specify what happens if the robot needs to switch to a different task and stop the current one. If at time $t$, the robot has to infer the processes needed to execute a task, it may be that for some process the starting action start $t_{\pi}$ or the ending action $e n d_{\pi}$ are not derivable; namely, $\mathcal{A} \cup \mathcal{C} \not \exists \exists \mathbf{x}, t \operatorname{Poss}\left(\operatorname{start}_{\pi}(\mathbf{x}, t), \sigma\right)$. To modify the current execution and make the activation of a process possible, it is desirable to obtain an explanation for enabling a specific action $a$. This is the formula $\psi \in \mathcal{L}$, such that:

$$
\mathcal{A} \cup \mathcal{C} \cup\{\psi\} \not \models \text {, and } \mathcal{A} \cup \mathcal{C} \cup\{\psi\} \models \exists \mathbf{x}, t \operatorname{Poss}\left(\operatorname{start}_{\pi}(\mathbf{x}, t), \sigma\right),
$$

where the proviso $\mathcal{A} \cup \mathcal{C} \cup\{\psi\} \not$ means that the explanation added to the theory does not imply the contradiction. Explanations can be computed using first-order abduction (see, e.g., Marquis [1991] and Cialdea and Pirri [1993]). Here, $\mathcal{A}$ is the action theory, $\mathcal{C}$ is the set of temporal-spatial constraints, and $\psi$ is the formula explaining the observation $\exists \mathbf{x}, t \operatorname{Poss}\left(\operatorname{start}_{\pi}(\mathbf{x}, t), \sigma\right)$; that is, $\psi$ is the formula to be added to ensure the inference.

Examples of such processes are reported by Finzi and Pirri [2005, 2010], Carbone et al. [2008], Pirri and Reiter [2000], and Pirri [2011] in the framework of Situation Calculus [Pirri and Reiter 1999]. However, processes are defined in several other frameworks, as, for example, in STRIPS, Graphplan, and PDDL [Blum and Langford 
1999; McDermott 2003; Fox and Long 2003; Fikes and Nilsson 1971]. Each of these has its own advantages in terms of expressive power, computational feasibility, and constraints expressible in the language. When robot planning requires the orchestration of all components according to temporal constraints, the planning system usually takes advantage of temporal networks to manage temporal constraints [Allen 1983; Dechter et al. 1991; Vilain et al. 1986; Block et al. 2006; Morris et al. 2001]. Here, we use Situation Calculus to model high-level control of robot processes because of its expressive power both for managing parallel processes and time-space constraints and for defining interference and reconfiguration cost. The stimuli and stimulus-response model proposed in this work could be built on any action theory that handles robot components, processes, and constraints and is able to express robot mental state at a precise time, together with all cause-effect relations affected by the response. This need is clarified in the next section, where we compute the cost of an explanation.

\section{TO SWITCH OR NOT TO SWITCH}

We are now at a point in the formalization of the stimulus-response where we can illustrate how a decision can be taken about whether to respond to a stimulus. To illustrate the decision problem, we continue with the WiFi disconnection example.

Example 6.1. The robot is executing task $\tau_{10}$, expecting to approach victims in a rescue area. At time $t$, the process $\pi_{W i F i}$ get disconnected, $h_{\beta}\left(\mathbf{z}_{W i F i}\right)=1$; hence $\mathcal{E}\left(\mathbf{x}_{W i F i}, t\right)=1$. Therefore, the action $e n d_{W i F i}$ becomes possible, and process $\pi_{W i F i}$ is interrupted. From table $\mathbf{A}$, the robot is advised to switch to task $\tau_{4}$, which corresponds to recovery WiFi signal, so it can choose between continuing the current task without the WiFi signal or switching to task $\tau_{4}$. To make the switch, it has to discontinue several active processes in $\tau_{10}$ (see the Table VII in Appendix A), and it has to activate only process $\pi_{1, C_{11}}$, which is read access to missing data, because all others required processes are already active in $\tau_{10}$. However, the constraints of the kind $\pi_{i} \mathbf{o p}_{k} \pi_{j}$ in the processes have changed, and they need to be recomputed in the new task $\tau_{4}$. Therefore, the system has to rate the effort and risks that its choice implies.

Here, we assume that before any decision to switch is taken, the response tasks to switch to has been selected according to the scores given by matrix $\mathbf{A}$ (see Section 4). We first consider the switching cost, given the best scored task to switch to. The cost to switch from the current to a new task breaks down into a reconfiguration and an interference cost with respect to a specific task. To these costs we may add a second reconfiguration cost if we need to return to the current task.

Reconfiguration cost: This is the cost to reconfigure the current robot state, which amounts to activating new processes $\pi_{R_{1}}, \ldots, \pi_{R_{m}}$. More precisely, the reconfiguration cost calculates the effort the system has to invest to infer all the transition and precondition axioms that are required to make processes $\pi_{R_{1}}, \ldots, \pi_{R_{m}}$ executable by the robot at the current state.

Interference cost: This is the cost needed to terminate active processes $\pi_{S_{1}}, \ldots, \pi_{S_{k}}$ specifying the current task, which are either not needed or would hinder the newly chosen task.

Let $\tau_{i}$ be the current task under execution, and let $\tau_{j}$ be a chosen task. Let $\Pi_{R}=$ $\left\{\pi_{R_{1}}, \ldots, \pi_{R_{m}}\right\}$ be all the processes that need to be activated to operate task $\tau_{j}$ and which are not yet active, and let $\Pi_{S}=\left\{\pi_{S_{1}}, \ldots, \pi_{S_{k}}\right\}$ be all the active processes that need to be turned off. Then, according to Equation (21), there is a set of formulas $\Psi$ and a set of formula $\Phi$ that must be inferred to explain, respectively, how processes $\Pi_{R}$ can be 
activated and how processes $\Pi_{S}$ can be turned off:

$$
\begin{aligned}
& \mathcal{A} \cup \mathcal{C} \cup \Psi \models \exists \mathbf{x}, t \bigwedge_{i=1}^{m} \operatorname{Poss}\left(\operatorname{start}_{\pi_{R_{i}}}(\mathbf{x}, t), \sigma\right) \wedge \bigwedge_{i \neq j} \pi_{R_{i}} \mathbf{o p} \pi_{R_{j}}\left[\mathbb{s}_{i}, \Phi_{j}\right], \quad \forall \pi_{R_{i}} \in \Pi_{R} \\
& \mathcal{A} \cup \mathcal{C} \cup \Phi=\exists \mathbf{x}, t \bigwedge_{i=1}^{k} \operatorname{Poss}\left(\operatorname{end}_{\pi_{S_{i}}}(\mathbf{x}, t), \sigma\right) \wedge \bigwedge_{i \neq j} \pi_{S_{i}} \mathbf{o p} \pi_{S_{j}}\left[\Phi_{i}, \Phi_{j}\right], \quad \forall \pi_{S_{i}} \in \Pi_{S} .
\end{aligned}
$$

Note that $\Psi$ and $\Phi$ are not necessarily minimal explanations (see Marquis [1991] and Cialdea and Pirri [1993] for the minimality problem in computing first-order explanations), but we might assume here that these explanations nevertheless minimize the action sequences.

The cost of reconfiguration and interference amounts to computing the cost of these statements. We define the cost considering first the terms of the language; namely, variables and functions of any arity. We exclude from the cost evaluation the terms mentioning variables of sort situation and variables of sort action: For example do( $a, s)$ is excluded, but not $d o\left(A(\mathbf{x}), S_{0}\right)$ since $S_{0}$ is a constant of sort situation and the action term $A$ is not a variable. Let $\eta$ be a term of the language; the cost of $\eta$ is inductively given by the function $v$, defined as follows:

$$
v(\eta)= \begin{cases}0 & \text { if } \eta \text { does not mention terms of sort action } \\ 1 & \text { if } \eta=\operatorname{start}_{\pi}(\mathbf{x}, t) \text { or } \eta=e n d_{\pi}(\mathbf{x}, t) \\ \sum_{i=1}^{k} v\left(A_{i}\right) & \text { if } \eta \text { mentions } A_{1}, \ldots, A_{k} \text { terms of sort action }\end{cases}
$$

Here, $t$ is a term of sort time and $\mathbf{x}$ is a list of terms (i.e., variables, constants, functions of any arity) of any sort. Now, to extend the cost computation to formulas, we consider the following restrictions. Let $\varphi \in \mathcal{L}$; we assume that $\varphi$ either does not mention terms of sort action and situation or, if it does mention them, these are in the form $d o\left(\left[A_{1}, \ldots, A_{m}\right]\right)$, with none of the action terms $A_{i}$ as variables. Then, let $\operatorname{DNF}(\varphi)=\bar{Q} \mathbf{x}\left[C_{1}\left(\mathbf{x}_{1}\right) \vee \cdots \vee C_{m}\left(\mathbf{x}_{m}\right)\right] \equiv \varphi$ be the equivalent transformation of $\varphi$ into DNF; namely, into disjunctive normal form in which each clause $C_{i}\left(\mathbf{x}_{i}\right), 1 \leq i \leq m$, is a conjunction of literals, with $\mathbf{x}_{i}$ terms varying on all sorts. Variables mentioned in $\mathbf{x}_{i}$ are included in $\mathbf{x}$ and bound by the quantifiers matrix $\bar{Q} \mathbf{x}$. Clearly, any variable $x$ appearing in $\mathbf{x}_{i}$ can neither be of sort action nor situation. Then the cost of the formula $\varphi$ is inductively defined as follows:

$$
\operatorname{cost}(\varphi)= \begin{cases}v(\mathbf{x}) & \text { if } \varphi=L(\mathbf{x}) \text { is a literal } \\ \sum_{i} \operatorname{cost}\left(L_{i}\left(\mathbf{x}_{i}\right)\right) & \text { if } \varphi \equiv \bar{Q} \mathbf{x}\left(L_{1}\left(\mathbf{x}_{1}\right) \wedge \cdots \wedge L_{k}\left(\mathbf{x}_{k}\right)\right) \\ \min \left\{\operatorname{cost}\left(C_{i}\left(\mathbf{x}_{i}\right)\right)\right\} & \text { if } \varphi \equiv \bar{Q} \mathbf{x}\left(C_{1}\left(\mathbf{x}_{1}\right) \vee \cdots \vee C_{m}\left(\mathbf{x}_{m}\right)\right)\end{cases}
$$

Note that if $\varphi$ is a tautology, then $\varphi$ can be reduced to a tautology-namely $\varphi \equiv$ $B \vee \neg B \vee C$, for any clause $C$. Hence, $\min \{\operatorname{cost}(\varphi)\}=0$, since $B$ does not mention terms of sort action. On the other hand, if $\varphi$ is a contradiction, then $\varphi \equiv B \wedge \neg B \wedge \bigwedge_{i} \psi_{i}$ for any conjunction $\bigwedge_{i} \psi_{i}$ of literals of $\mathcal{L}$. Hence, $\operatorname{cost}(\varphi)=\min \left\{\sum_{i} \operatorname{cost}\left(L_{i}\right)\right\}$, with $\sum_{i} \operatorname{cost}\left(L_{i}\right)$ tending to infinity since we may take any literal from the language and with any sequence of actions as argument. Finally, given a set of formulas $\Psi$, the cost of $\Psi$ is the sum of the costs of each $\psi \in \Psi$.

We are now ready to determine the cost for inferring the explanations needed to activate a preferred task $\tau_{j}$ in terms of the cost of the preconditions and constraints for $\tau_{j}$, at time $t$, according to Equation (22) and in terms of discontinuing a number of processes occurring in the current task $\tau_{i}$. Given Equation (22), the cost of inference for switching is:

$$
\operatorname{switch} \operatorname{Cost}\left(\left\langle\tau_{j}, \tau_{i}\right\rangle, t\right)=\operatorname{cost}(\Psi, t)+\operatorname{cost}(\Phi, t)+J \cos t\left(\Psi^{\prime}, t^{\prime}\right), \quad t^{\prime}>t, q \in\{0,1\} .
$$




\begin{tabular}{|c||r|r|r|r|}
\cline { 2 - 5 } \multicolumn{1}{c|}{} & processes & $\pi_{1, C_{2}}$ & $\pi_{1, C_{11}}$ & $\pi_{1, C_{13}}$ \\
\cline { 2 - 5 } \multicolumn{1}{c|}{} & yields & $y_{\pi_{1}, C_{2}}$ & $y_{\pi_{1}, C_{11}}$ & $y_{\pi_{1}, C_{13}}$ \\
\hline max scored tasks & \multicolumn{3}{|c}{} \\
\hline$\tau_{4}$ & & .24 & & \\
\hline$\tau_{6}$ & & & $\mathbf{3 2}$ & \\
\hline$\tau_{3}$ & & & & $\mathbf{. 4 8}$ \\
\hline
\end{tabular}

Fig. 3. The switching costs for the task $\tau_{12}$, data retrieval (see Table VII ). The up-rows indicate the processes the task is assembled from and their yields. In the left column are the tasks that obtained the maximum switching score in score matrix A. The gray cells indicate the average normalized switching cost to the preferred task, indicated in the left column.

Here, $\Psi^{\prime}$ is like $\Psi$, but it is the cost of returning to the current task after the task the robot has switched to is completed. If $J=0$, there is no need to return to the current task $\tau_{i}$.

Having obtained the switching cost and knowing the response task for the triggered stimulus, we can compute the payoff. Here, the payoff is simply the culmination of the stimulus-response problem and it is, indeed, the payoff for switching. Let $R_{S}$ be a function specifying the choice of switching and $R_{I}$ the analogous function for inhibition. Then the payoff for switching is simply $R_{S}-R_{I}$.

Obviously, we expect to attribute the switching cost to $R_{S}$. However, if the switching cost is computed before any decision is taken, then this is like attributing the cost to both $R_{S}$ and $R_{I}$. In fact, by its definition, computing the switching cost is the same as abducing the set of explanations needed to activate the new task and deactivating the current state; thus, it follows that this inference process ends up weighting the decision well before the decision is taken. ${ }^{2}$

Therefore, to both correctly define the two functions and to avoid burdening the decision, the switching cost can be replaced by a prediction of it. To predict the switching cost, as defined in Equation (25), two steps are needed:

(1) Define a trend of the switching cost according to time, in terms of the sequence of actions entering the $\sigma \mathrm{s}$ of the two logical implications in Equation (22).

(2) Produce an estimate of the switching cost for different time durations of the current task.

To this end, we generate a number of action sequences for as many time lapses within a time range bounded by 120 minutes. For each of these samples, the cost given in Equation (25) is computed. An example of the averages of the normalized switching cost for task $\tau_{12}$-namely, data retrieval -is given in Figure 3 with respect to each of the response tasks to the stimuli that can be triggered by each of the processes composing the execution of task $\tau_{12}$.

The trends of the switching cost for a task are established with respect to each of its processes and for each of the response tasks to the stimuli of its process yields; therefore, it is defined for each pair $\left\langle\tau_{i}, \tau_{j}\right\rangle$. Once the trends are obtained, the switching cost is approximated by its frequencies with respect to time.

To estimate the cost distribution, we define a bivariate random variable $\mathbf{X}$ taking values in the domain Times $\times$ SwitchingCost for each pair $\left\langle\tau_{i}, \tau_{j}\right\rangle$. The realization of the bivariate variable $\mathbf{X}$ is computed by a nonparametric density $f_{X}$ measuring the switching cost frequencies to discontinue the current task $\tau_{i}$ and activate task $\tau_{j}$. Here, we use a Gaussian kernel to estimate kernal density, as we did in Section 3; here, however, we have a bivariate estimator (time and cost), so we define the kernel density

\footnotetext{
${ }^{2}$ Computing the explanations for switching can be viewed as the intellectual work that the system performs, as opposed to the practical and physical work of executing tasks.
} 


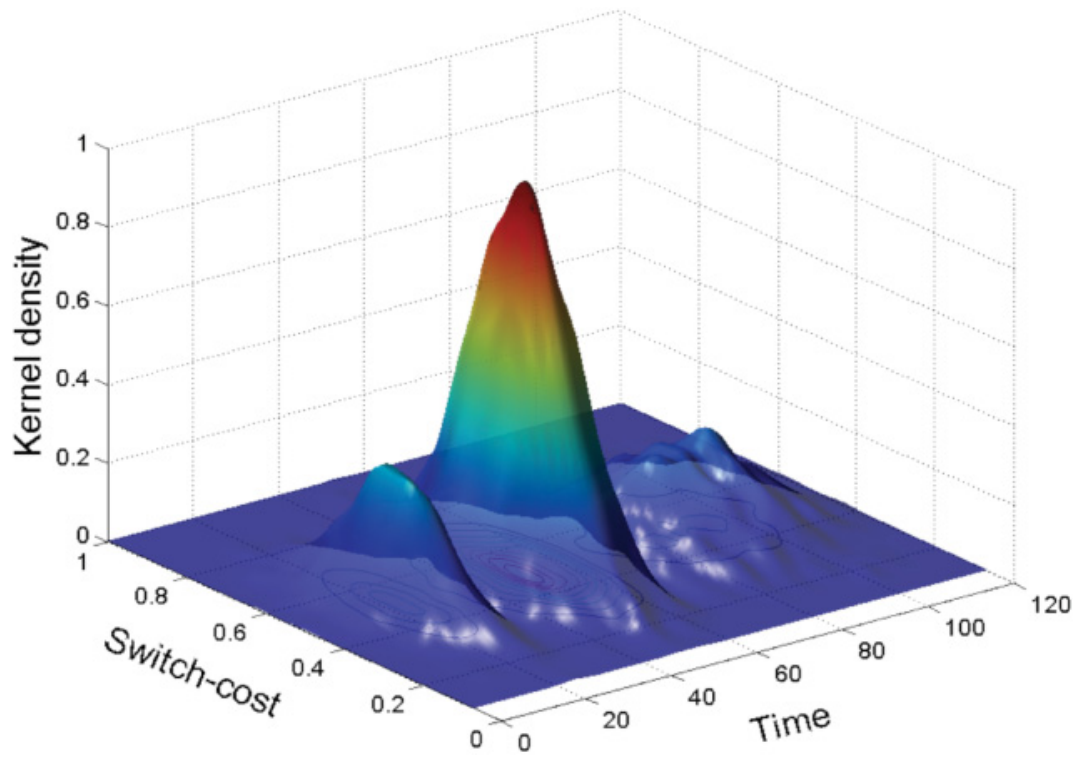

Fig. 4. Kernel density $f_{X}$ for switching cost from the task $\tau_{12}$ to task $\tau_{4}$, showing frequencies of switching cost in a time lapse of 120 minutes, taken with respect to 10 cost trends.

for a generic task as follows:

$$
f_{X}(\mathbf{x})=\frac{1}{n} \sum_{i=1}^{n} \frac{1}{\operatorname{det}(\mathbf{H})} K\left(\operatorname{diag}\left(\mathbf{H}^{-1}\right)^{\top}\left(\mathbf{x}-\mathbf{X}_{i}\right)\right) .
$$

Here, $K(v)=\frac{1}{\sqrt{2 \pi}} \exp \left(-\frac{1}{2} v^{2}\right)$ and $\mathbf{H}=n^{-1 / 6} \Sigma^{1 / 2}$, where $\Sigma$ is the diagonal covariance matrix of the samples; namely, $\mathbf{H}$ follows the Scott's rule of thumb, and $\operatorname{diag}(\cdot)$ indicates the diagonal. The grid has resolution $\mathbf{H}$, and the bivariate $\mathbf{X}_{i}$ is built using samples from the switch cost trend taken at time $t$ for different action sequences. The switching cost density for tasks $\left(\tau_{12}, \tau_{4}\right)$ is illustrated in Figure 4 , where time is presented in minutes. To compute the modes of kernel density, we proceed as in Section 3. Let $v_{i}=\operatorname{diag}\left(\mathbf{H}^{-1}\right)^{\top}\left(\mathbf{x}-\mathbf{X}_{i}\right)$ and $\operatorname{diag}(\mathbf{H})=\left(h_{1}, h_{2}\right)^{\top}$; then the gradient of $f_{X}$ is:

$$
\begin{aligned}
\nabla f_{X}\left(x_{1}, x_{2}\right)= & \left\{\frac{1}{n} \sum_{i=1}^{n}-\frac{1}{h_{j} \operatorname{det}(\mathbf{H})} K\left(v_{i}\right) v_{i}\right\}_{j=1,2}=\left\{-f_{X}\left(x_{1}, x_{2}\right)\left(\frac{x_{1}}{h_{j}^{2}}+\frac{x_{2}}{\operatorname{det}(\mathbf{H})}\right)\right. \\
& \left.+\frac{1}{n} \sum_{i=1}^{n} \frac{1}{\operatorname{det}(\mathbf{H})} K\left(u_{i}\right)\left(\frac{X_{i 1}}{h_{j}^{2}}+\frac{X_{i 2}}{\operatorname{det}(\mathbf{H})}\right)\right\}_{j=1,2}
\end{aligned}
$$

As in Section 3, the modes $\widehat{\mathbf{x}}$ are collected by gradient ascent. The modes return the best prediction of the bivariate $\mathbf{x}$. Where the probability of $\mathbf{x}$ is below a threshold $\epsilon$ or where the probability is high but the estimated switching cost (given time $t$ ) is beyond the effort the robot system can afford, then we introduce a time failure. These failures, that can be collected for each pair $\left\langle\tau_{i}, \tau_{j}\right\rangle$, are best explained with an example.

Example 6.2. Continuing Example 6.1, consider the pair $\left(\tau_{10}, \tau_{4}\right)$ at time $t=72$. According to the cost trends, the nonparametric function $f_{X}(\mathbf{x})$ is drawn, and it has modes at $T=(35,71,110,119)$. At these times, the cost rates are $(0.3,0.5,0.7,0.9)$. Let $\epsilon=0.6$ be the maximum cost the robot can afford to switch. Since $t=72$, it follows 
that the cost can be afforded. If $t$ were 110 , then the rate would be $0.7>\epsilon$; hence, we would obtain a failure in the cost computation.

We collect the time to process failure by establishing the switching cost between $\left\langle\tau_{i}, \tau_{j}\right\rangle$ from the modes $\widehat{\mathbf{x}}$ of $f_{X}(\mathbf{x})$ as in the following set, where sc is switching_cost, and $\epsilon$ is the threshold for the effort the robot can afford:

$$
\left\{t_{i} \mid\left(t_{i}, s c_{i}\right) \in \widehat{\mathbf{x}}, f_{X}(\widehat{\mathbf{x}})<10^{-2} \vee s c_{i}>\epsilon\right\}
$$

Time to failure of the switching cost amounts to the belief that, at the specific time, either the effort of computing the state update is not affordable, or the switching cost value cannot be predicted. This time to failure is independent of any previous decision. Therefore, its best approximation is the exponential distribution with a constant hazard function; that is, it has no burn-in cost. Let $U_{s c}$ be a random variable with exponential distribution, with failure rate $^{3} \alpha=1 / \theta^{\prime}$ :

$$
\begin{aligned}
& F_{U_{s c}}(z)=1-\exp (-\alpha z), \quad C D F \\
& f_{U_{s c}}(z)=\alpha \exp -(\alpha z), \quad P D F .
\end{aligned}
$$

The reliability for time to failure of the process establishing the switching cost is $R_{U_{s c}}(t)=1-F_{U_{s c}}(t)=\exp (-\alpha t)$.

At this point, we consider the time to failure of the current tasks $\tau_{i}$ and the chosen task $\tau_{j}$. To estimate a task's time to failure, we introduce a random variable $W_{\tau}$ whose probability distribution indicates beliefs about the likelihood of failures time. The random variable $W_{\tau}$ is characterized by a Weibull distribution with parameters $(\beta, \theta)$, with $\theta$ the average time to failure and $\beta$ the failure rate ${ }^{4}$ (decreasing if $0<\beta \leq 1$ and increasing for $1<\beta<3.5$ ):

$$
\begin{aligned}
& F_{W_{\tau}}(z ; \beta, \theta)=1-\exp \left(-\left(\frac{z}{\theta}\right)^{\beta}\right), \quad C D F \\
& f_{W_{\tau}}(z ; \beta, \theta)=\frac{\beta \exp \left(-\left(\frac{z}{\theta}\right)^{\beta}\right)\left(\frac{z}{\theta}\right)^{\beta-1}}{\theta}, \quad P D F .
\end{aligned}
$$

Here, $\beta$ is increasing, $1<\beta \leq 3.5$, and $\theta$ ranges between 0 and 120 minutes. Then, the reliability of task $\tau_{i}$ is $R_{W_{\tau_{i}}}(t)=P\left(W_{\tau_{i}}>t\right)=\exp \left(-\left(\frac{t}{\theta}\right)^{\beta}\right)$ at $t$; namely, the probability that the task will continue without failures for time $t<W_{\tau_{i}} \leq t_{\text {end }}$, needed to wrap up. For the reliability of the task sequence $\left\langle\tau_{i}, \tau_{j}\right\rangle$, we consider the reliability function $R_{\left\langle W_{\tau_{i}}, W_{\tau_{j}}\right\rangle}(t)$ :

$$
\begin{aligned}
R_{\left\langle W_{\tau_{i}}, W_{\tau_{j}}\right\rangle}(t) & =P\left(W_{\tau_{j}}>t\right) P\left(W_{\tau_{i}}>t\right)=\left(1-F_{W_{\tau_{i}}}\left(t ; \beta_{1}, \theta_{1}\right)\right)\left(1-F_{W_{\tau_{j}}}\left(t ; \beta_{2}, \theta_{2}\right)\right) \\
& =\exp \left(-\sum_{i \in\{1,2\}}\left(\frac{t}{\theta_{i}}\right)^{\beta_{i}}\right)
\end{aligned}
$$

This expression can be extended to three tasks in sequence.

\footnotetext{
${ }^{3}$ Here, $\alpha$ indicates the failure rate and differs from the denotation of confidence level given in Section 3.

${ }^{4}$ Here, $\beta$ denotes the rate parameter of the Weibull distribution and differs from the parameters used in Section 3. Note that if $\beta=1$, then the Weibull reduces to the exponential distribution.
} 

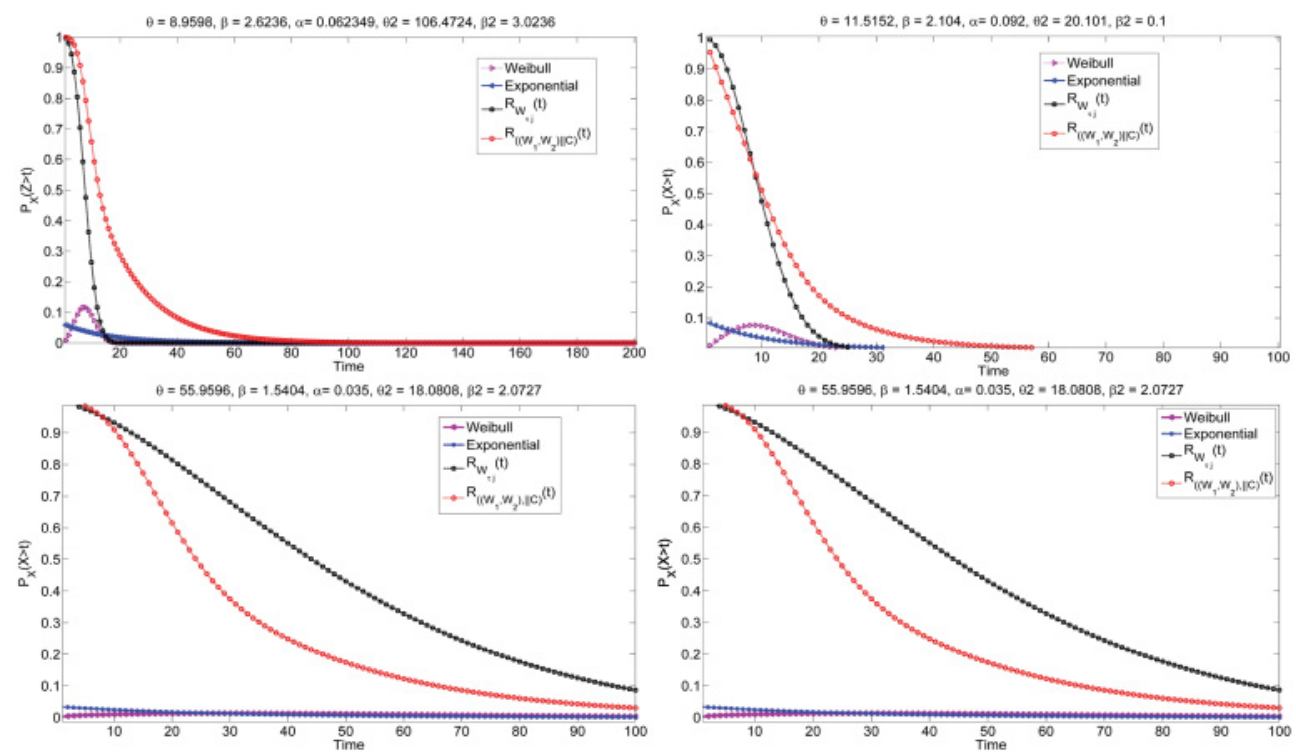

Fig. 5. Examples of reliability of the current task (the dashed black line) against the reliability of the parallel composition of the switching cost reliability with the sequence formed by the current task $\tau_{i}$ and the chosen task $\tau_{j}$.

Finally, the reliability of the combination of the two tasks, taken in sequence, and the switching cost estimation, taken in parallel with the two tasks, is defined as follows:

$$
\begin{aligned}
R_{\left(\left\langle W_{\tau_{i}}, W_{\tau_{j}}\right\rangle \| U_{s c}\right)}(t) & =P\left(W_{\tau_{j}}>t\right) P\left(W_{\tau_{i}}>t\right)+P\left(U_{s c}>t\right)-P\left(W_{\tau_{j}}>t\right) P\left(W_{\tau_{i}}>t\right) P\left(U_{s c}>t\right) \\
& =\exp (-t \alpha)+\exp \left(-\sum_{i \in\{1,2\}}\left(\frac{t}{\theta_{i}}\right)^{\beta_{i}}\right)-\exp \left(-t \alpha-\sum_{i \in\{1,2\}}\left(\frac{t}{\theta_{i}}\right)^{\beta_{i}}\right) .
\end{aligned}
$$

At this point, we can see that $R_{\left(\left\langle W_{\tau_{i}}, W_{\tau_{j}}\right\rangle \| U_{s c}\right)}(t)$ and $R_{W_{\tau_{i}}}(t)$ are the two sought for functions $R_{S}$ and $R_{I}$ introduced earlier, and, therefore, the payoff for switching is:

$$
\text { payoff }=R_{\left(\left\langle W_{\tau_{i}}, W_{\tau_{j}}\right\rangle \| U_{s c}\right)}(t)-R_{W_{\tau_{i}}}(t) .
$$

Clearly, if at the given time $t, R_{W_{\tau_{i}}}(t)<R_{\left(\left\langle W_{\tau_{i}}, W_{\tau_{j}}\right\rangle \| U_{s c}\right)}(t)$, then the payoff is positive and switching is convenient; otherwise it is negative or null. Some examples showing reliability are illustrated in Figure 5. The simulation-based computation for task time to failures is reported in the Appendix A.7, and experiments computing the payoff for switching are reported in Section 7.3.

\section{EXPERIMENTS}

In this section, we present the results of experiments undertaken to evaluate the proposed framework's performance. In Section 7.1, the performance of the stimulus model is tested. In Section 7.2, we evaluate the generalizability of the score matrix $\mathbf{A}$, described in Section 4. In Section 7.3, we test the switching decision described in Section 6. The whole framework is tested in Section 7.4. Finally, in Section 7.5, we compare the performance of the stimulus-response framework with two different approaches to robot replanning [Beetz et al. 2010; Talamadupula et al. 2010; Göbelbecker et al. 2011]. For all experiments, we recruited 20 operators between 26 and 32 years of age, in good 


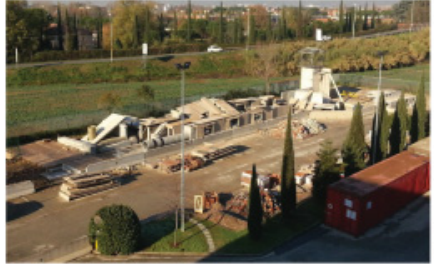

(a)

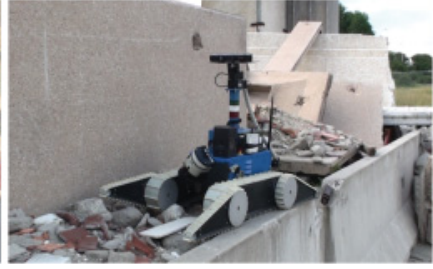

(b)

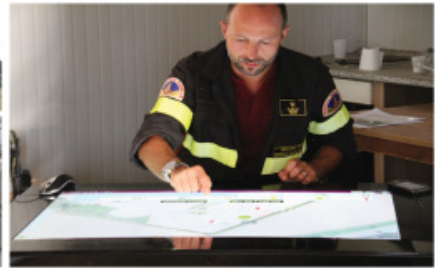

(c)

Fig. 6. (a) Simulated disaster area. (b) Obstacles located to the disaster area. (c) Operator interacting with the robot through the task switching interface.

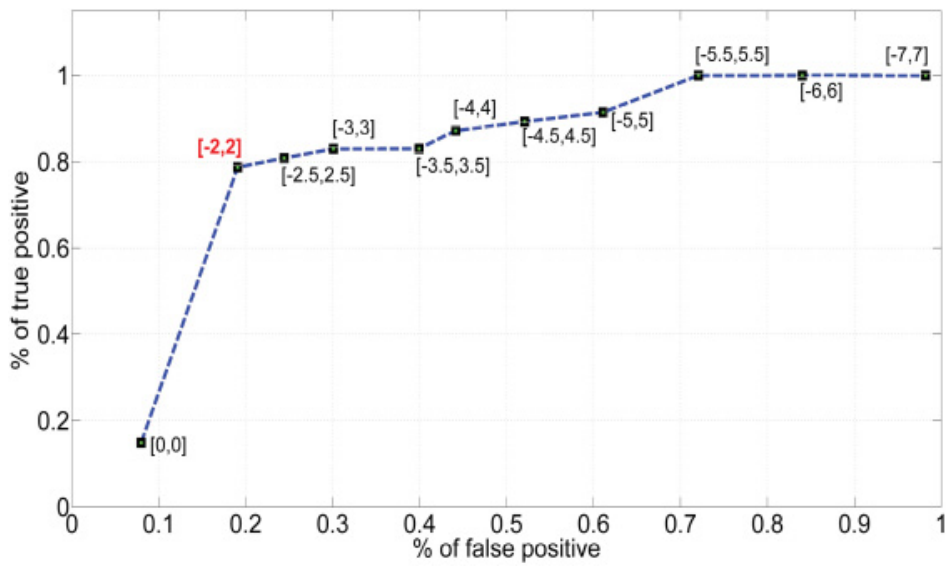

Fig. 7. Performance of the stimulus model: ROC curve of the true and false positives as the time range, indicated in brackets, changes.

physical conditions. Each operator performed up to 10 trials using the task switching interface (see Figure 6(c)) and choosing a task from the task library described in Section A.3, in Appendix A.

\subsection{Performance of the Stimulus Model}

The performance of the stimulus model is evaluated by the ROC curve of both the true and false stimuli over a varying time range, with the stimulus occurring in the range. The performance of the model is measured on a testset, collected as follows. Once a task is chosen, the operators manually label the yields of the running processes upon the occurrence of a stimulus. The yields, labeled by the operators, were compared with the results of the stimuli classification provided by the model (Section 3). Both correct classifications and mismatches are noted, considering the reference time interval [init, end] centered at the time the operator notified occurrences of stimuli. Figure 7 shows the ROC curve of the stimulus model with respect to the size of $\Delta t$. The trend reports the percentage of true positives versus false positives for every considered time range. Then, $[-2,2]$ is taken as the reference time interval to evaluate the correctness of the stimulus model, due to the tradeoff between the false and true positives.

\subsection{Generalizability of the Score Matrix A}

The generalizability of score matrix $\mathbf{A}$ is measured according to the convergence of the Mean Square Error (MSE) with respect to two different values of rank $d$ of the matrices $\mathbf{Q}$ and $\mathbf{P}$, factorizing $\mathbf{A}$ (Section 4). The dataset for both stimuli and responses 


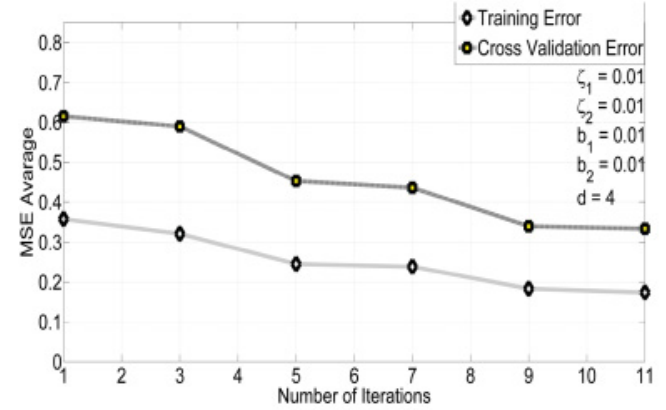

(a) $d=4$

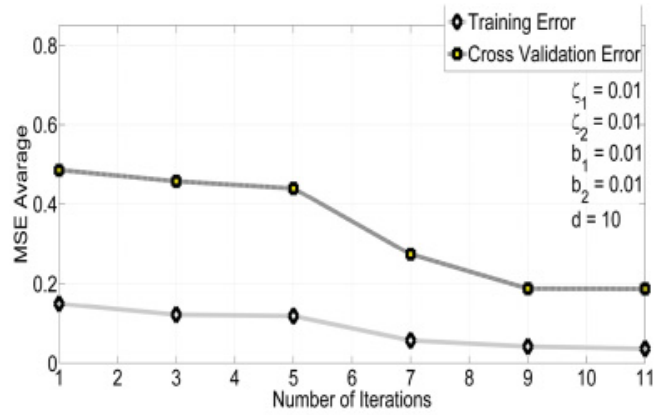

(b) $d=10$

Fig. 8. Learning curve for the score matrix $\mathbf{A}$ given two different rank values of the matrices $\mathbf{Q}$ and $\mathbf{P}$, factorizing A: (a) $d=4$ and (b) $d=10$. The curve is derived with a $k$-fold cross-validation technique.

to them is gathered by operators in the field as they execute tasks chosen from the task library via the task switching interface set to active mode (see Section A.5 in Appendix A). The operator responds to a stimulus by either switching or continuinginhibiting the stimulus. A $k$-fold cross-validation technique on the gathered dataset is used to assess the generalizability of the model. Figures 8(a) and 8(b) show the average of both the MSE training and cross-validation error under two different values $(d=4$ and $d=10$, respectively) specifying the rank of the matrices $\mathbf{Q}$ and $\mathbf{P}$. Indeed, $d=10$ is the reference value of the rank. The completed score matrix, resulting from the crossvalidation process, is given in Tables XI and XII in Appendix A. The MSE error with respect to the training set is shown in Figure 2.

\subsection{Reliability Tests and Payoff}

This experiment computes the payoff of switching, taking into account both the time to failure for each task and the time to failure for the switching cost, as described in Section 6 (see Table XIII in Appendix A). Table III lists both the values of the reliability $R_{W_{\tau_{i}}}(t)$ of the tasks that the robot was executing before the occurrence of the stimulus and the reliability $R_{\left\langle\left\langle W_{\tau_{j}}, W_{\tau_{i}}\right\rangle \mid U_{s c}\right\rangle}(t)$ of the combination of the current tasks with the tasks to switch to. The indices of the tasks in Table III are made explicit in Table VII in Appendix A. The choice of switching, given the payoff, is indicated in the last column. These data are used in Section 7.4 to evaluate the whole stimulus-response framework.

\subsection{Experimental Evaluation for Task Switching}

In this experiment, we compare system decisions with operator decisions. The set of robot tasks are variations of task $\tau_{10}$; namely, approaching a number of objects located in the experiment area and already reported in the 3D map. A number of stimuli are purposefully triggered: battery exhaustion, $\mathrm{WiFi}$ disconnection, write access to full memory, and read access to missing data. During execution, robot choices along with payoff values and the log for reliability computation are recorded (see Section 6). In parallel, the responses selected by the operator via the task-switching interface are recorded. Note that the operator does not have to choose the preferred task from the score matrix $\mathbf{A}$, as the robot will do. Furthermore, the operator does not see the robot's choices, to ensure unbiased decisions. The gathered data were used to build the sequences of task choices for both the robot and the human operators. In Figure 9, we report one run, among all those collected, showing operator and robot choices in parallel. Comparing these sequences, we note that in more than $90 \%$ of the cases the model of task switching correctly induces the robot to shift to the same task as that 
Table III. Reliability Examples Used in Experiment 7.4

\begin{tabular}{|c|c|c|c|c|c|}
\hline Time & Current Task & Candidate Task & $R_{W_{\tau_{i}}}(t)$ & $R_{\left\langle\left\langle W_{\tau_{i}}, W_{\tau_{j}}\right\rangle \| U_{s c}\right\rangle}(t)$ & Task Switching \\
\hline 11 & $\tau_{10}$ & $\tau_{4}$ & .3065 & .4150 & Yes \\
\hline 22 & $\tau_{10}$ & $\tau_{6}$ & .3375 & .4387 & Yes \\
\hline 34 & $\tau_{10}$ & $\tau_{12}$ & .4571 & .4705 & Yes \\
\hline 45 & $\tau_{10}$ & $\tau_{5}$ & .3917 & .4955 & Yes \\
\hline 70 & $\tau_{10}$ & $\tau_{4}$ & .2736 & .3272 & Yes \\
\hline 76 & $\tau_{4}$ & $\tau_{12}$ & .3018 & .6043 & Yes \\
\hline 89 & $\tau_{10}$ & $\tau_{6}$ & .4181 & .5361 & Yes \\
\hline 103 & $\tau_{10}$ & $\tau_{12}$ & .3894 & .2840 & Yes \\
\hline 124 & $\tau_{10}$ & $\tau_{5}$ & .5083 & .6735 & Yes \\
\hline 134 & $\tau_{5}$ & $\tau_{4}$ & .5310 & .5649 & Yes \\
\hline 158 & $\tau_{10}$ & $\tau_{12}$ & .2739 & .3770 & Yes \\
\hline 164 & $\tau_{12}$ & $\tau_{4}$ & .3366 & .5213 & Yes \\
\hline 179 & $\tau_{6}$ & $\tau_{4}$ & .3861 & .6015 & Yes \\
\hline 202 & $\tau_{10}$ & $\tau_{5}$ & .2991 & .3127 & Yes \\
\hline 217 & $\tau_{5}$ & $\tau_{4}$ & .3305 & .3820 & Yes \\
\hline 246 & $\tau_{10}$ & $\tau_{12}$ & .5937 & .6436 & Yes \\
\hline 273 & $\tau_{10}$ & $\tau_{4}$ & .4031 & .4036 & Yes \\
\hline 302 & $\tau_{10}$ & $\tau_{6}$ & .3593 & .5912 & Yes \\
\hline 321 & $\tau_{10}$ & $\tau_{5}$ & .2859 & .3683 & Yes \\
\hline 344 & $\tau_{10}$ & $\tau_{12}$ & .2039 & .3091 & Yes \\
\hline 366 & $\tau_{10}$ & $\tau_{4}$ & .3709 & .5905 & Yes \\
\hline 395 & $\tau_{10}$ & $\tau_{5}$ & .1901 & .2197 & Yes \\
\hline
\end{tabular}

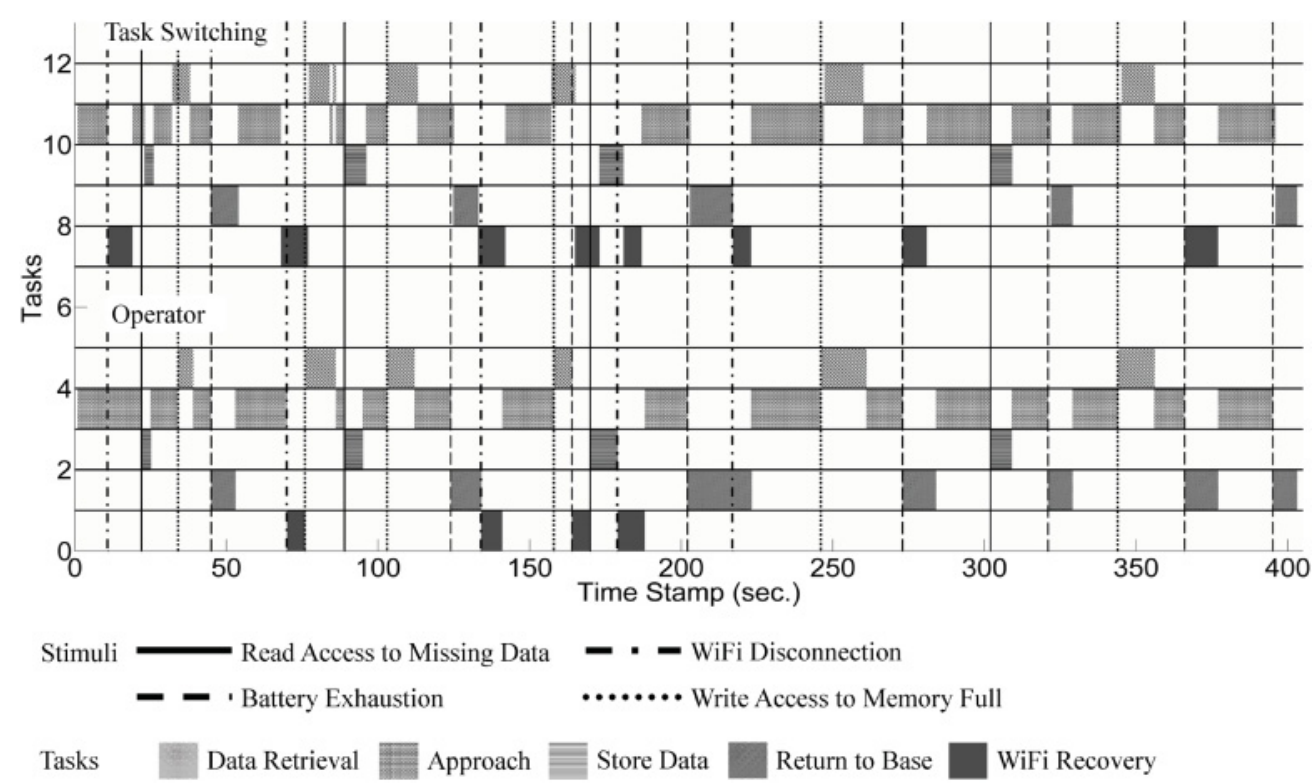

Fig. 9. Sequences of task choices according to both the robot and the human operator, performed during the mission of approaching selected objects positioned in known places in the experiment area. Vertical lines denote the occurrences of the stimuli at different times. In more than $90 \%$ of the cases, task switching correctly induces the robot to shift to the same task as that selected by the operator. In more than $41.1 \%$ of cases, the robot anticipates the operator in shifting. At time $t=11 \mathrm{sec}$., as well as at time $t=217 \mathrm{sec}$., the operator inhibits the stimuli while the robot switches to a new task. 
selected by the operator. Among the percentage of correct robot switches, $41.1 \%$ happen in advance. At time $t=11 \mathrm{sec}$., as well as at time $t=217 \mathrm{sec}$., the operator inhibits the WiFi disconnection stimulus, while the model induces the robot to switch to the signal task recovery.

\subsection{Comparison with Robot Replanning}

For this experiment, we considered two approaches to robot replanning. The first [Beetz et al. 2010] is based on the implementation of execution-monitoring (CRAM-EM) in the CRAM plan language. In this implementation, stimuli are explicitly modeled either as intrinsicStateChangeEvents or as physicalEvents. Each of these events is associated with a recovery procedure. The execution-monitoring determines whether the occurrence of an event can affect the successful outcome of the execution of the current plan. In such a case, the monitor generates a new plan on the basis of the set of predefined failure recovery procedures whose execution is intended to restore the state of the task at hand. The second case [Talamadupula et al. 2010; Göbelbecker et al. 2011] is based on the implementation of a deterministic action goal-oriented POMDP (DAGO-POMDP) in Decision Theoretic PDDL, in which all actions have no-zero cost, and an optimal policy can be formulated as a finite horizon contingent plan. Stimuli are modeled as perceptual propositions (i.e., percepts). Tasks are associated with stimuli by suitably assigning either high-value rewards or low-value costs to the actions needed to execute the tasks. In this implementation, plans are possibly rebuilt online to react to changes in the underlying domain (e.g., when goals are modified or when stimuli occur). Details about the implementation of both approaches are out of the scope of this work. The experiments were performed in a real partially destroyed area (see Figure 6(a)), where robot can overcome some of the obstacles (see Figure 6(b)).

The performance of the model of task switching with respect to both CRAM-EM and DAGO-POMDP replanning approaches was evaluated by measuring the following indexes: (1) the time average of reaction to stimuli (MSRT); (2) the time average of response to stimuli (MRST); the average time of mission accomplishment (MMA); (4) the ratio between the number of switches performed by the task switching model and the number of replanning instances, where the response to stimuli provided by all the models is the same (SWRPC); (5) the ratio between the number of switches and the number of replanning instances, where responses are different from each other (SWRPD); and, finally, (6) the ratio between the number of times the task switching model inhibited the stimulus and the number of times both CRAM-EM and DAGOPOMDP decided to replan (IRP).

MSRT measures the average time elapsed between the occurrence of a stimulus and its identification. For CRAM-EM, this time corresponds to the average time elapsed between the occurrence of the stimulus and the detection of the corresponding event. For DAGO-POMDP, this is the average time elapsed between the occurrence of the stimulus and the decision to rebuild the plan in reaction to it. MRST represents, on average, the time needed to select the best response to the stimulus (e.g., shifting or continuing). For both replanning approaches, this index denotes the average time spent for replanning. To measure these indexes, the experiments were set up as follows. The robot is instructed to autonomously approach an object whose position is known on the given $3 \mathrm{D}$ map. The time estimated to accomplish the task is about 20 min (this is a large area). The task ends when either the estimated time expires or the robot reaches all the targets. The occurrence of a stimulus on WiFi signal quality and on battery power level is simulated after $6 \mathrm{~min}$. and after $12 \mathrm{~min}$. from the beginning of the task, respectively. The signal associated with a stimulus is kept up for $2 \mathrm{~min}$. The pulse of the signal has a trapezoidal profile. The choice of a reduced number of stimuli is motivated by the fact that we want to bound the domain of both different approaches 
Table IV. Time Comparison with Robot Replanning

\begin{tabular}{|c||c|c|c|}
\hline \hline Model & MSRT (sec.) & MRST (sec.) & MMA (sec.) \\
\hline Stimulus-Response & 51.49 & 88.17 & 837.86 \\
\hline CRAM-EM & 70.94 & 159.03 & 1093.72 \\
\hline DAGO-POMDP & 69.22 & 131.07 & 961.91 \\
\hline
\end{tabular}

Table V. Performance Comparison with Robot Replanning

\begin{tabular}{|c||c|c|c|}
\hline \hline Model & SWRPC & SWRPD & IRP \\
\hline \hline CRAM-EM & .45 & .30 & .25 \\
\hline DAGO-POMDP & .60 & .15 & .25 \\
\hline
\end{tabular}

to replanning. This guarantees the applicability of the two approaches to replanning. Both the duration and the profile of the pulse associated with a stimulus have been chosen to test the degree of proactivity of the stimulus-response, but also to allow both CRAM-EM and DAGO-POMDP to infer plans that avoid stuck conditions for the robot. On the other hand, the static setting of the environment allows us to make repeatable trials. In this setting, 20 trials were performed.

Table IV reports the results obtained from measuring the time indexes MSRT, MRST, and MMA. The MSRT index of the task switching model is lower than the indexes of the other models. The main reason is that the stimulus model anticipates the identification of the stimuli (see Section 3). Instead, both CRAM-EM and DAGO-POMDP require that the amplitude of the signal associated with a stimulus reaches a certain threshold in order to detect the stimulus. Both CRAM-EM and DAGO-POMDP perform a state space search to generate new plans in the replanning phase, whereas the task switching model use the payoff model to select the best choice for a new task (see Section 6), so no state space search is required. Therefore, the MRST time index of the proposed model is the lowest.

The task switching model demonstrated better performance in terms of time needed to accomplish the mission. In fact, the model can compromise between brave and conservative behavior, which is not allowed in a purely reactive framework. The proposed model is able to inhibit the stimulus, and thus balance continuous changes by preserving focus on the task at hand if switching is too demanding. This behavior outperforms the purely reactive behavior of both CRAM-EM and DAGO-POMDP. The SWRPC, SWRPD, and IRP indexes reported in Table V validate these considerations, also showing that the robot not only behaves more rationally but also more rapidly in accomplishing a mission.

In addition, the indexes MSRT, MRST, and MMA highlight that the system is more flexible than both CRAM-EM and DAGO-POMDP. The first column of Table V shows that the robot chooses to switch only in the $45 \%$ and $60 \%$ of cases in which the other two models decided to replan. On the other hand, the values of the indexes in the second column of Table V show that the task selected, when switching is the choice, are different; this is due to the lack of a stimulus-response matrix learned from humans in the other two models. Therefore, replanning in both CRAM-EM and DAGO-POMDP is wired into the behavior and not learned. Finally, the values of the IRP index in the third column of Table V show the ability of the task switching model to be conservative with respect to the task at hand.

These considerations prove that the task switching model, by exploiting both highlevel reasoning and learning from operators, effectively harmonizes robot choices with context requests by conjugating a rational behavior with a flexible one. 


\section{CONCLUSION}

In this work, we proposed a model for robot stimuli and stimulus-response that can significantly improve robot control in general, and particularly in the robot's interaction with the environment. The proposed approach indicates a new way to address robot cognitive control by showing that interaction with the environment can be proactive if stimuli are taken into account not individually but as a whole system whose specific aspects can be learned by interacting with human users. This gained flexibility in robot control can increase the usability of the robot, especially for operators who have to deal with it in extreme environments like first-responder training areas or even post-disaster environments.

Our approach is completely novel, and, to our knowledge, no other approach to robot cognitive control has developed these ideas. The proposed method is supported by a full implementation in an advanced robot platform endowed with multiple abilities to perform several tasks, in which the interplay with operators plays a crucial role for both learning and evaluating performance. Our approach is inspired by the wide psychophysical and psychological literature on human patterns in stimulus-response and task-switching, although we have provided an integrated model that is tailored for a robot system and is replicable for other robotic platforms.

Only recently has the need to model the stimulus-response pattern leading to task switching and the cognitive functions of shifting and inhibition become a hot topic in cognitive robotics [Medina Ayala et al. 2012; Nakano et al. 2011; Ganesh and Burdet 2013; Ajoudani et al. 2013; Ding and Fang 2013; Tao et al. 2012; Li et al. 2013; Sung et al. 2013; Jamone et al. 2013; Saglam and Byl 2013; and Li and Cheah 2012]. The earliest studies were carried out within brain-actuated interaction [Milln et al. 2004], mechatronics [Capi 2007], behavior learning [Ito et al. 2006], navigation [Althaus and Christensen 2003], and planning [Finzi and Pirri 2005]. Recently, several studies posited the need to model task switching to cope with adaptivity and ecological behaviors in a dynamic environment [Capi et al. 2008; Suzuki et al. 2009; Wawerla and Vaughan 2009; Durkee et al. 2011; D’Ambrosio et al. 2011].

In building the stimulus model, the main difficulties we encountered were due to the experimental setting and data collection from several operators. This led us to provide an outliers rejection method specifically for the stimuli; this method supports the selection of the training set on the basis of the features manifold eliciting the stimuli. This turned out to be a good solution to transfer training data acquired by human operators to the robot training set. To learn the stimulus-response selection, we still used several operators, but to infer unknown data we use low-rank matrix factorization methods [Zhang et al. 2012; Chen 2008; Buchanan and Fitzgibbon 2005; Canny 2002] widely employed in recommendation systems and collaborative filtering [Ko and Lee 2002; L. Herlocker et al. 2004]). This model has proved suitable to estimate the latent parameters underlying stimulus-response mapping, as well as to predict the weights given by each stimulus to each response, which are missing in the observed data.

The problem of shifting and inhibition induced by stimuli was modeled by a switching cost, obtained by conjugating a cost to reconfigure the robot state for the new goal and the cost to resolve interference with the current activities set. Finally, the decision problem culminates in the definition of the payoff for switching, which requires us to consider the risk and effort in computing the cost. We used time to failure to combine the whole effort and predict the reliability of all the decision components to calculate the payoff for switching.

We also introduced a connection between stimuli and reasoning level by defining a boundary shared by the two levels via the mapping $\mathcal{E}$ that plays the role of transduction, lifting the terms from the sample space of features, where yields are sampled, to the 
terms of the language. Thus, logical inference provides the grounds for the causal and constraint relations among processes carrying the information driven by stimuli. The logical structure is also the framework within which both processes and the robot's mental states can be defined, and it is where the reconfiguration and interference costs can be fully interpreted.

The proposed method improves both autonomy and flexibility in robot control. Furthermore, according to the experiments done to verify the coherence and rationality of robot behaviors with respect to operator behavior while teleoperating the robot, the approach proves to be very promising for improving the proactive interactions with the environment required by a robot relating to people and their needs.

Comparing the proposed framework with two other approaches to robot replanning, both robot flexibility and performance, in terms of time and tasks choices, are improved. It follows that robot usability also is improved since user stress can be reduced by limiting the workload that continuous monitoring of robot activities demands of the operator.

\section{A. APPENDIX: IMPLEMENTATION}

\section{A.1. Hardware Description}

The robotic platform is designed for harsh, unmanned environments. These are largescale environments where robot missions last for long time periods and several events and stimuli burden the robot's exploration and reporting activities.

The robot platform (c)BlueBotics, named Absolem, has a central body where the electronics is located and has two bogies on the sides. Each bogie is made of a central track for locomotion and two active flippers on both ends to extend the climbing capabilities. A breakable passive differential system allows the rotation of the bogies around the body. Several sensors are installed on the platform, among them a rotating $2 \mathrm{D}$ laser scanner, an omnidirectional camera with a 360-degree field of view, an IMU/GPS, and a panoramic microphone.

\section{A.2. Low-Level Robot Functionalities}

The robot's Simultaneous Localization and Mapping (SLAM) is based on a real-time 3D ICP [Pomerleau et al. 2013]. A Dead Reckoning Navigation System (DRNS), based on a Complementary Filter (CF), estimates the Euler angles of the robot body from the fusion of both odometry and the IMU inertial data [Cao et al. 2009; Kubelka and Reinstein 2012]. Both ICP-based SLAM and DRNS provide, at real-time, the pose of the robot as a feedback to the Trajectory Tracking Controller (TTC) [Gianni et al. 2013b]. Static traversability cost assessment of the ground, from point cloud data, is performed by both physics-based terrain [Stoyanov et al. 2010; Papadakis and Pirri 2012] and physical constraints analysis [Cafaro et al. 2013]. Visual perception of the environment is limited to object (e.g., signs, cars, and persons) detection accomplished with an omnidirectional camera [Hurych et al. 2011].

\section{A.3. Robot Model}

The robot model provides a representation of both the hardware and software components and of the processes activated by each component. For example, the SLAM software module is modeled as a component, whereas the mapping and localization functionalities are represented as processes managed by the SLAM component. Table VI lists the main components and processes of the robot system model. Here, by 
Table VI. Components and Processes of the Robot Model

\begin{tabular}{|c|c|c|c|}
\hline Component Id & Component Description & Process Id & Process Description \\
\hline$C_{1}$ & Battery & $\pi_{1, C_{1}}$ & Power Level Monitoring \\
\hline$C_{2}$ & WiFi & $\pi_{1, C_{2}}$ & Connection Management \\
\hline \multirow{3}{*}{$C_{3}$} & \multirow{3}{*}{ 3D Laser Scan } & $\pi_{1, C_{3}}$ & ROS Drivers Running \\
\hline & & $\pi_{2, C_{3}}$ & Laser Rotating \\
\hline & & $\pi_{3, C_{3}}$ & Scanning \\
\hline \multirow{4}{*}{$C_{4}$} & \multirow{4}{*}{ SLAM } & $\pi_{1, C_{4}}$ & ROS Packages Running \\
\hline & & $\pi_{2, C_{4}}$ & 2D Mapping \\
\hline & & $\pi_{3, C_{4}}$ & 3D Mapping \\
\hline & & $\pi_{4, C_{4}}$ & Localizing \\
\hline \multirow{3}{*}{$C_{5}$} & \multirow{3}{*}{ Vision System } & $\pi_{1, C_{5}}$ & ROS Drivers Running \\
\hline & & $\pi_{2, C_{5}}$ & Calibrating \\
\hline & & $\pi_{3, C_{5}}$ & Streaming \\
\hline \multirow{3}{*}{$C_{6}$} & \multirow{3}{*}{ Visual Perception } & $\pi_{1, C_{6}}$ & ROS Packages Running \\
\hline & & $\pi_{2, C_{6}}$ & Detecting Object/Person \\
\hline & & $\pi_{3, C_{6}}$ & Recognizing Object/Person \\
\hline \multirow{4}{*}{$C_{7}$} & \multirow{4}{*}{ Robot Engine } & $\pi_{1, C_{7}}$ & ROS Drivers Running \\
\hline & & $\pi_{2, C_{7}}$ & Locomotion System Monitoring \\
\hline & & $\pi_{3, C_{7}}$ & Temperature Monitoring \\
\hline & & $\pi_{4, C_{7}}$ & Locking Differential Drive \\
\hline \multirow{3}{*}{$C_{8}$} & \multirow{3}{*}{ Trajectory Control } & $\pi_{1, C_{8}}$ & ROS Packages Running \\
\hline & & $\pi_{2, C_{8}}$ & Planning \\
\hline & & $\pi_{3, C_{8}}$ & Tracking \\
\hline \multirow{2}{*}{$C_{9}$} & \multirow[t]{2}{*}{ Dialogue } & $\pi_{1, C_{9}}$ & Human to Robot Exchanging msg \\
\hline & & $\pi_{2, C_{9}}$ & Robot to Human Exchanging msg \\
\hline \multirow{3}{*}{$C_{10}$} & \multirow{3}{*}{ Microphone } & $\pi_{1, C_{10}}$ & ROS Drivers Running \\
\hline & & $\pi_{2, C_{10}}$ & Hearing \\
\hline & & $\pi_{3, C_{10}}$ & Speech Recognizing \\
\hline \multirow{3}{*}{$C_{11}$} & \multirow{3}{*}{ Memory } & $\pi_{1, C_{11}}$ & Data Reading \\
\hline & & $\pi_{2, C_{11}}$ & Data Writing \\
\hline & & $\pi_{3, C_{11}}$ & Memory Checking \\
\hline \multirow{2}{*}{$C_{12}$} & \multirow[t]{2}{*}{ IMU } & $\pi_{1, C_{12}}$ & ROS Drivers Running \\
\hline & & $\pi_{2, C_{12}}$ & Robot Pose Estimating \\
\hline$C_{13}$ & ROS Master Core & $\pi_{1, C_{13}}$ & ROS Core Running \\
\hline \multirow{2}{*}{$C_{14}$} & \multirow[t]{2}{*}{ Central Processing Unit } & $\pi_{1, C_{14}}$ & Payload Monitoring \\
\hline & & $\pi_{2, C_{14}}$ & Temperature Monitoring \\
\hline \multirow{5}{*}{$C_{15}$} & \multirow{5}{*}{ Terrain Analysis } & $\pi_{1, C_{15}}$ & ROS Packages Running \\
\hline & & $\pi_{2, C_{15}}$ & Traversability Analysis \\
\hline & & $\pi_{3, C_{15}}$ & Ground Subtracting \& Analysis \\
\hline & & $\pi_{4, C_{15}}$ & Obstacle Detecting \\
\hline & & $\pi_{5, C_{15}}$ & Adapting Robot Morphology \\
\hline \multirow{2}{*}{$C_{16}$} & \multirow[t]{2}{*}{ Diagnostic } & $\pi_{1, C_{16}}$ & System Check \\
\hline & & $\pi_{2, C_{16}}$ & Repair \\
\hline
\end{tabular}

$C_{k}$ we denote the $k$-th robot component and by $\pi_{j, C_{k}}$ the $j$-th process managed by the $k$-th component.

A set of robot processes defines a task. This set of tasks forms the robot task library. For example, the task to overcome an obstacle requires that all the processes managed by the 3D Laser Scan component are active, that the 3D mapping process is running, and that the process adapting robot morphology to the terrain provides the correct 
positions for the flippers. Table VII lists the set of tasks designed in agreement with the available robot functionalities. Here, by $\tau_{i}$ we denote the $i$-th task in the task library. The right column in Table VII lists all the processes that need to be activated for the robot to perform the corresponding task.

Each process yields a quantum of information with characteristic features. This quantum of information is called the yield of the process. For example, the process monitoring the temperature of the robot engine outputs, over time, the servo-motors temperature. The information carried by the processes regulates the robot's behaviors. For example, the engine temperature could reach a critical level and damage the robot servo-motors. The critical engine temperature level, as well as the loss of the WiFi connection between the robot and the remote command post, are stimuli, and they are identified from the process yields. Table VIII lists a description for each robot stimuli. Note that each process can trigger a single stimulus; therefore, the number and types of stimuli are determined by the number and types of processes and these, in turn, are determined by the number and types of robot components. In the table, the term $y_{\pi_{j, C_{k}}}$ denotes the stimulus triggered by the yield of the $j$-th process managed by the $k$-th component $C_{k}$.

\section{A.4. Robot Control}

Robot control is defined by a declarative temporal model of its activities and a planning engine. The declarative temporal model is specified in the Temporal Flexible Situation Calculus (TFSC) [Finzi and Pirri 2005; Carbone et al. 2008] so that the main components and processes of the robot system reported in Table VI are represented by cause-effect relationships as well as by the temporal and spatial constraints among the processes [Pirri and Reiter 2000; Finzi and Pirri 2005], as discussed in Section 5. Planning is composed of two main logical modules: the plan generator and the execution monitoring. The plan generator relies on a library of Prolog scripts that designates the set of tasks (see Table VII) that the robot can perform according to the specified processes, their temporal constraints (compatibilities), and their preconditions. Execution-monitoring is a continuous process ensuring that both the set of action sequences generated by the plan generator according to the TFSC model and the current state of the domain knowledge are consistently executed. The execution monitoring embeds the model of task switching to regulate the behavior of the robot in responses to the incoming stimuli. Both the TFSC model and the planning engine are implemented in Eclipse Prolog [Apt 2006], which optimally combines the power of a constraint solver (for time and compatibility constraints) with inference in order to generate the set of action sequences and also to enable continuous updates from incoming new knowledge by using finite regression [Pirri and Reiter 2000].

The distributed working memory structure interconnects the TFSC model and the planning engine with the task switching engine and with the robot functionalities that are integrated in the ROS Operating System (ROS) [Quigley et al. 2009] (see Figure 10). The structure is distributed over a collection of ROS services, organized as a set of addressable memory registers. In addition to the usual reading and writing operations, the structure provides additional operations like memory dumping and update acknowledgment operations, suitable to check both consistency and persistence of the state of the memory. The working memory system also takes care of mapping the internal state of the robot into a representation suitable for planning and reasoning about tasks.

The overall robot control provides bidirectional communication interfaces with the human user. It further allows the human operator to switch between several 


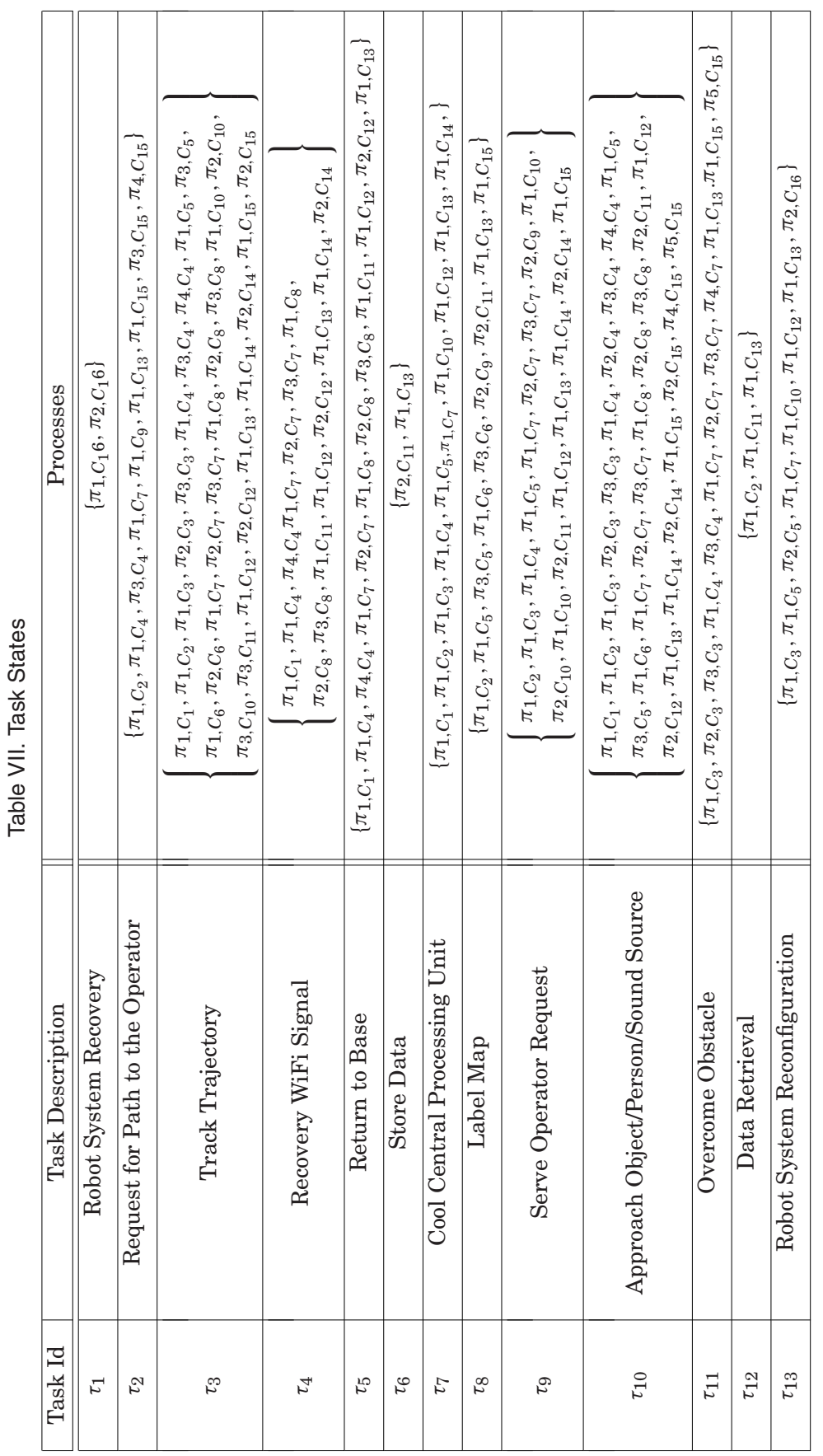




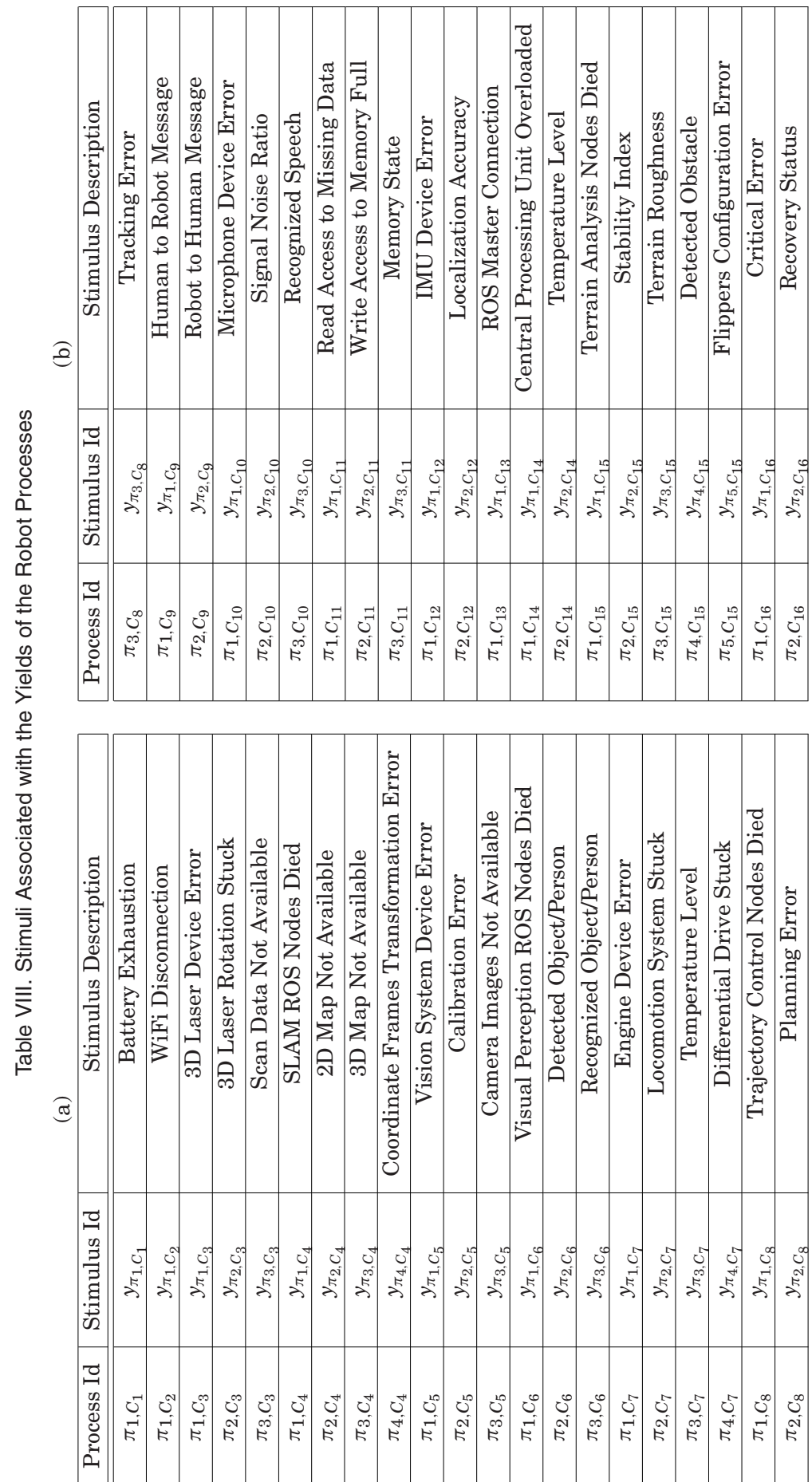




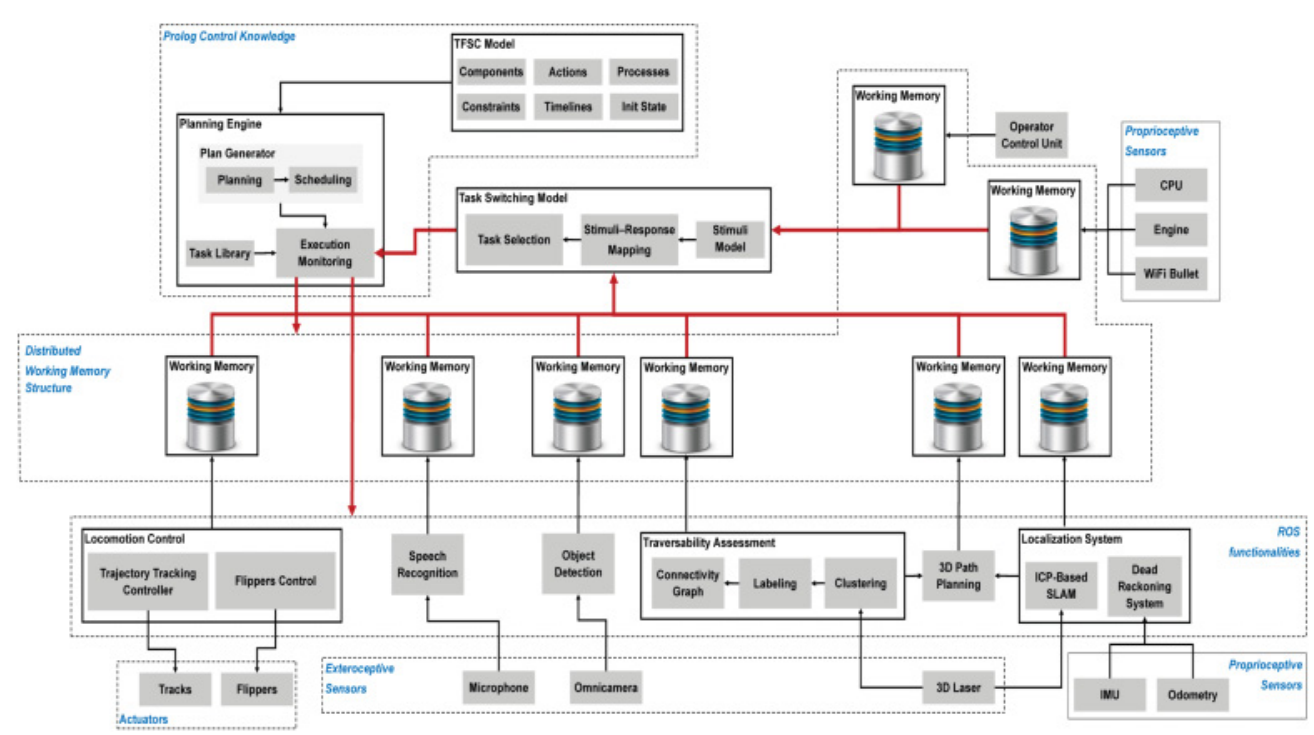

Fig. 10. Distributed working memory structure.

operational modalities that lie between autonomous and teleoperated modes during the execution of a task.

\section{A.5. Task Switching Learning Interface}

An interactive interface serves in dataset gathering (see Figure 11). The layout has four principal panels: (1) the stimulus panel, (2) the robot state panel, (3) the task state panel, and (4) the control panel. The stimulus panel includes vertical tabs listing robot components. Each tab is composed of a set of frames, each related to a component process and displaying the trend of the process and its yields. An additional button allows the operator to manually notify the robot of the occurrence of a stimulus. The operator can disable a frame via a check-box button. By selecting a tab in the stimulus panel, the operator can observe the state of each robot component, as well as the yields of the processes of that component. The robot state panel is composed of both a text box reporting the task the robot is currently accomplishing and a table listing all the running processes required to perform that task. The operator can indicate which task the robot has to perform via the tasks buttons displayed in the task panel. The control panel has two horizontal tabs: (1) the robot steering tab and (2) the flipper configuration tab. In the first tab, a number of control commands are listed for steering the robot. The latter serves to change flipper configuration and lock/unlock the differential drive to manually control the robot locomotion system. In addition, the main window reports both reconfiguration and interference costs. Three radio buttons set the interface for three different user interaction modalities: (1) passive mode, (2) active mode, and (3) acquisition mode. The passive mode allows the operator to manually notify the occurrence of the stimuli by observing the yields of the processes. The active mode enables a mixed robot-human initiative. In this modality, the interface displays the data yielded by the active processes and notifies the operator of the occurrence of a stimulus in real-time. Accordingly, the operator can select the task the robot has to perform or allow the robot to continue its current task. The acquisition mode gathers the active processes and shows the robot choice if a stimulus occurs. In this mode, the 


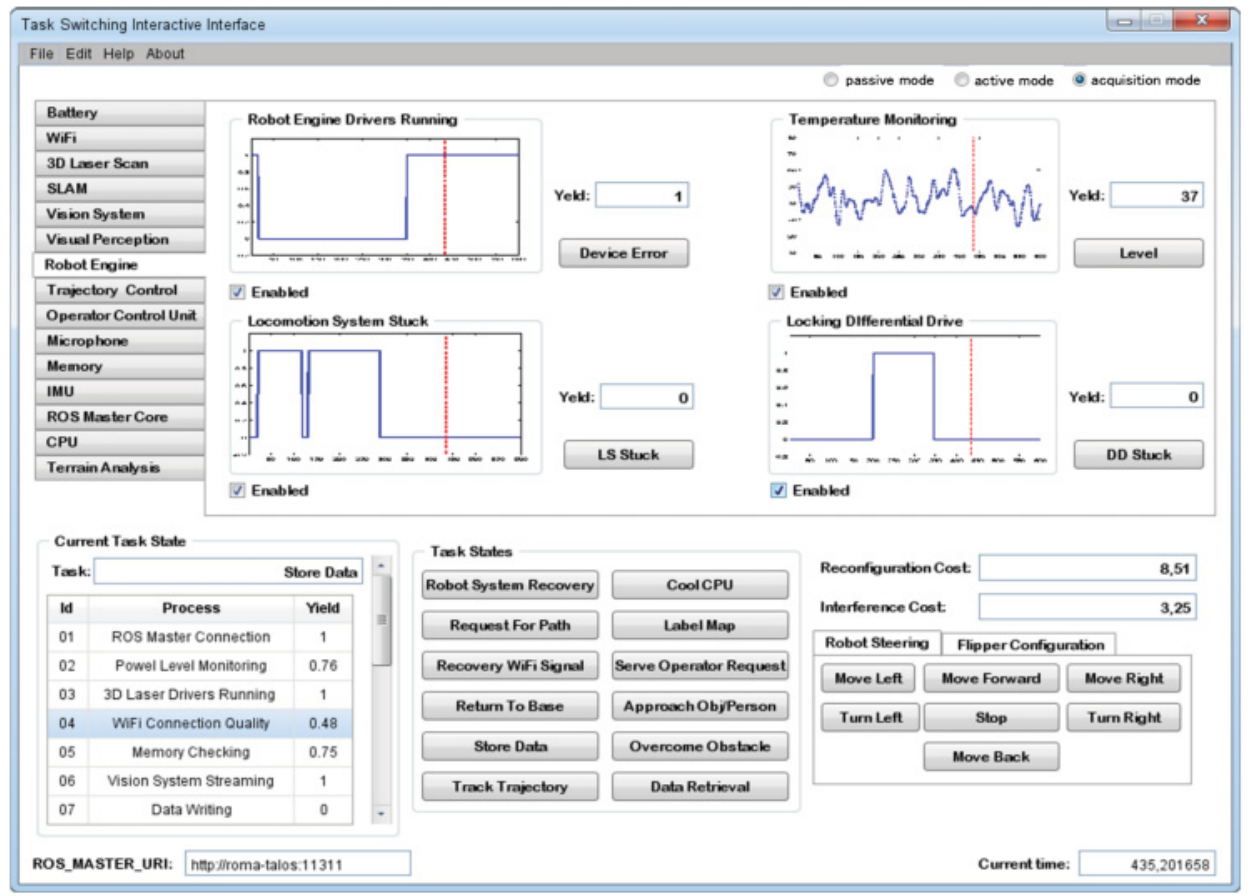

Fig. 11. Task switching interactive interface.

interface notifies the human operator of the occurrence of a stimulus, the choices taken by the robot to respond, and the task chosen; in this mode, the operator can store his or her choice for further analysis.

\section{A.6. Stimulus-Response Matrix: An Example}

Tables IX and X, read side by side, provide an example of the score matrix $\mathbf{A}$ introduced in Section 4 . Each entry $a_{i j}$ represents the score given by a stimulus $y_{\pi_{j, C_{k}}}$, triggered by the yield of the $j$-th process, managed by the $k$-th component, to a task $\tau_{i}$. This matrix is filled with data gathered from the experiments described in Section 4 using the graphical user interface described in Section A.5. Dashes denote those stimulus-task pairs that the operators never selected during the experiments. Tables XI and XII, read side by side, form the estimated score matrix $\mathbf{A}$.

\section{A.7. Task Failure Rates}

For each task listed in Table VII , failure rates have been computed within the simulation environment ARE [Gianni et al. 2013a]. Each task is assigned a time for termination, and, during the task execution, no stimuli occur. For each task, we repeat up to 10 trials. In the ARE environment, it is possible to generate several events populating the simulated environment; events blocking one of the running processes determine the failure of the task. In Table XIII, we report the time to failure for each task in the simulated environment: In the first column, we list the task identifier, in the second the execution time to end the task, in the third column the number of failures, in the fourth the trial at which the failure occurred, and, in the last, the time at which failure took place. 


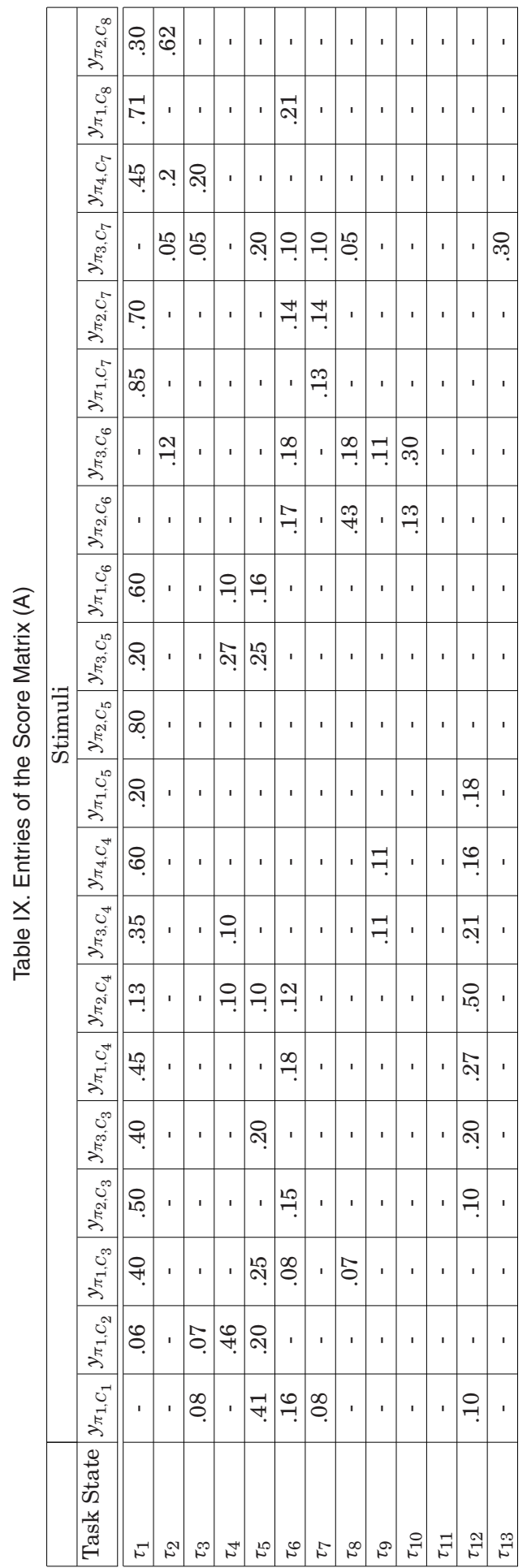




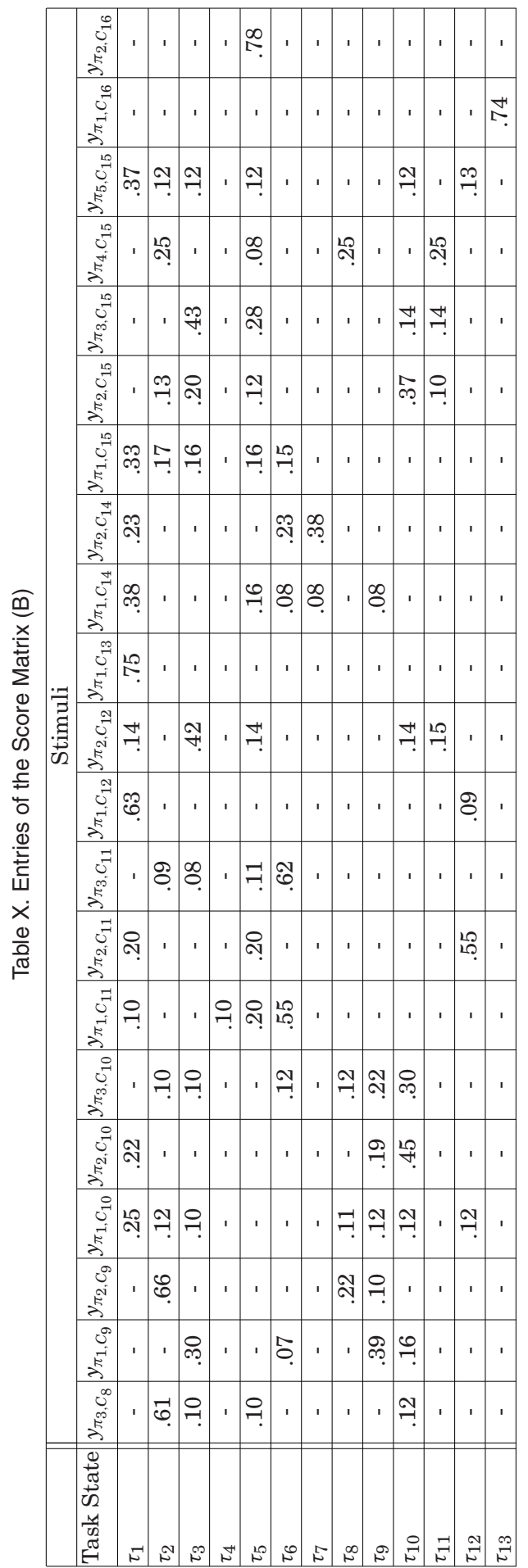




\begin{tabular}{|c|c|c|c|c|c|c|c|c|c|c|c|c|}
\hline & 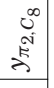 & ๑̊. & రิ & & & 60 & $\sigma$ & - & - & 0 & & 5. \\
\hline & 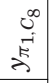 & ㄷ. & 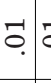 & 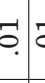 & a & & 0 & o. & 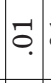 & & & 5.0 \\
\hline & 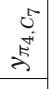 & L & คิ ฮ & จิ ฉ & ô. & 0.5 & $\sigma$ & o. & ô. & ș & & :- \\
\hline & $\begin{array}{c}5 \\
5 \\
s^{5} \\
\lambda^{2}\end{array}$ & ¿. & ? & & & $\stackrel{7}{\neg}$ & $\stackrel{0}{-}$ & $\stackrel{2}{0}$ & ô. & \% & ô & కై \\
\hline & $\begin{array}{r}5 \\
5 \\
5 \\
\underbrace{5} \\
\end{array}$ & พ & 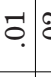 & $\stackrel{N}{0} \delta$ & 8 & 8 & $\stackrel{20}{\longrightarrow}$ & 0 & 0 & 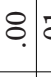 & 5 & 5.0 \\
\hline & $\begin{array}{c}5 \\
5 \\
5 \\
5 \\
\end{array}$ & ১. & 5 & b. & & 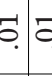 & 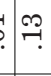 & 8 & - & 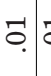 & & 5 \\
\hline & $\begin{array}{c}0 \\
c^{\circ} \\
s^{5} \\
z^{2}\end{array}$ & $\stackrel{7}{\circ}$ & 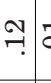 & $\bar{c}$ & 5 & ○. & 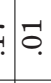 & $\neg$ & ๆี & ค़े & & ค. \\
\hline & $\begin{array}{c}0 \\
0 \\
\underbrace{0} \\
z^{2}\end{array}$ & 5. & 0 & os. & ô. & $\begin{array}{c}\delta \\
0\end{array}$ & $\%$ & 卖 & $\stackrel{\delta}{0}$ & $\stackrel{\oplus}{\rightarrow}$ & & 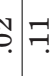 \\
\hline & 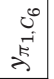 & ढ. & - & tot & 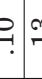 & $\because$ & \% & ó. & - & ô. & o. & 5. \\
\hline & $\begin{array}{c}50 \\
c^{10} \\
y^{5} \\
\end{array}$ & ন̣ & o. & $\begin{array}{l}\text { s. } \\
\text { o }\end{array}$ & ְ̦. & 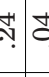 & 0 & ô. & 0 & ô. & ב & 5 \\
\hline 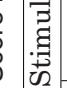 & 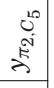 & 2 & oิ & co. & ô. & o. & ?. & 尺ิ. & $\stackrel{\Im}{o}$ & ô. & 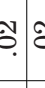 & vo \\
\hline & 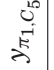 & શิ & $\stackrel{20}{0}$ & b. & 8 & 8, & $\%$ & 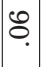 & 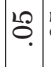 & 눙 & & $=0$ \\
\hline & 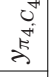 & $\bullet$ & 0 & 5 & 5 & 5 & of. & of & $F$ & ஜ. & 6 & 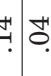 \\
\hline & $\begin{array}{c}c^{+1} \\
s^{\infty} \\
y^{2}\end{array}$ & 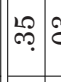 & $\%$ & 5 & $=8$ & b. & \% & - & $\exists$ & ô. & co. & र. \\
\hline & 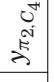 & $\stackrel{\rightarrow=}{0}$ & 0 & 5 & 이 & 이는? & 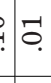 & ó. & - & 0. & 6 & \begin{tabular}{c} 
sit \\
\hdashline
\end{tabular} \\
\hline & $\begin{array}{r}0 \\
\overrightarrow{5} \\
\overrightarrow{5} \\
\end{array}$ & $\underset{:}{*}$ & 0 & 5 & 5 & 5 & - & - & o. & $\sigma$ & & : \\
\hline & $\begin{array}{c}\infty \\
c^{\infty} \\
y^{5} \\
5^{2}\end{array}$ & $\exists$ & 0 & 5 & of & จิ & o. & ô. & $\stackrel{\text { ô }}{0}$ & ô. & 6 & 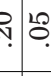 \\
\hline & $\begin{array}{c}c^{\infty} \\
\underbrace{5} \\
3^{2}\end{array}$ & \& & ๙̃. & ְ. & ठ্ & $\begin{array}{lll}0 \\
\end{array}$ & ำ & סิ. & 今 & ฮิ. & 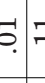 & $7: 18$ \\
\hline & $\begin{array}{l}0 \\
5 \\
5 \\
-5\end{array}$ & ๙ি. & 0 & $\overrightarrow{0}$ & o. & ণ़ & . & کิ. & $\begin{array}{c}\tilde{c} \\
0\end{array}$ & ô. & o. & 5.8 \\
\hline & $\begin{array}{l}5 \\
5 \\
5^{2} \\
\end{array}$ & $\stackrel{10}{\rightarrow}$ & so & 5 & 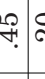 & จุ & ธุ. & - & 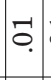 & 0 & & s. \\
\hline & $\begin{array}{c}5 \\
- \\
5 \\
-5\end{array}$ & ठ̣! & $\tilde{\delta}, q^{\alpha}$ & \&. & 8 & 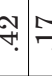 & $\overline{1}{ }^{\infty}$ & ơ & ô & ô. & 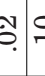 & 0 \\
\hline & 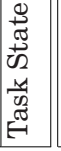 & $\vec{F}$ & $\mathbb{N}$ & $\stackrel{\sim}{\sim}$ & $\begin{array}{l}* \\
N^{\prime}\end{array}$ & 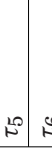 & 0 & $\infty$ & 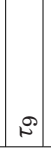 & 离 & $\vec{N}$ & \\
\hline
\end{tabular}




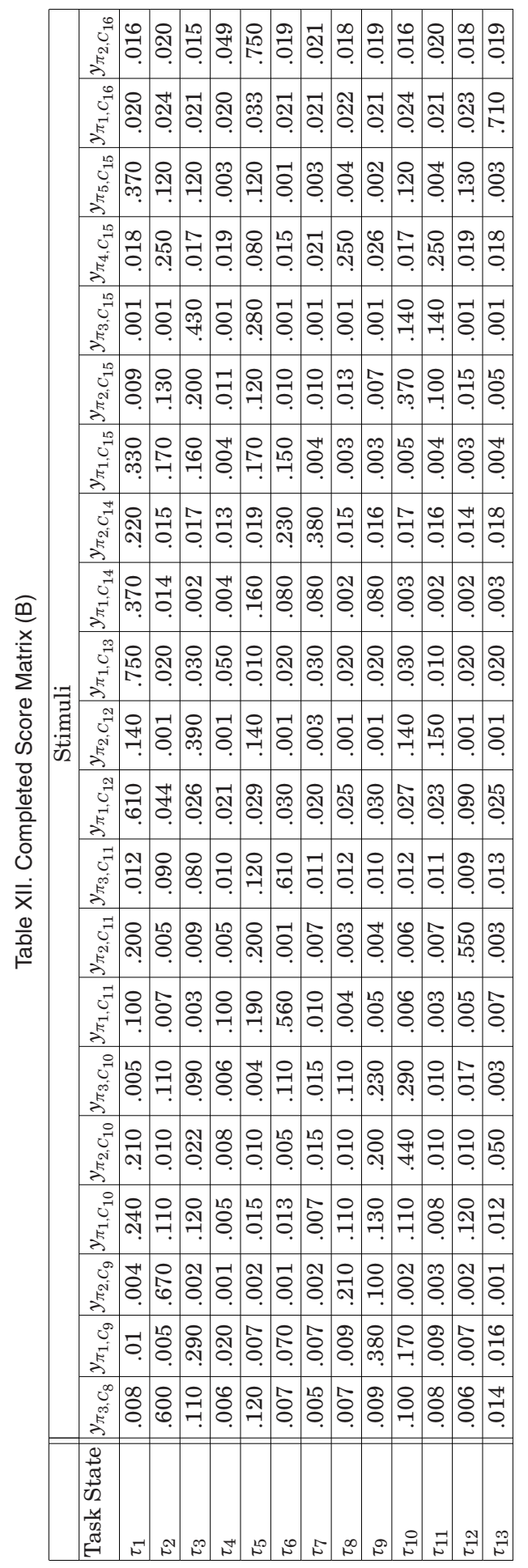


Table XIII. Task Time to Failure

\begin{tabular}{|c|c|c|c|c|}
\hline Task Id & Execution Time (min.) & \# of Failures & \multicolumn{2}{|c|}{ Failure time (min.) } \\
\hline$\tau_{1}$ & 18 & 0 & - & - \\
\hline$\tau_{2}$ & 16 & 1 & Trial 3 & 15.23 \\
\hline \multirow{4}{*}{$\tau_{3}$} & \multirow{4}{*}{46} & \multirow{4}{*}{4} & Trial 2 & 12.51 \\
\hline & & & Trial 3 & 28.09 \\
\hline & & & Trial 6 & 31.11 \\
\hline & & & Trial 9 & 5.06 \\
\hline \multirow{3}{*}{$\tau_{4}$} & \multirow{3}{*}{31} & \multirow{3}{*}{3} & Trial 4 & 19.01 \\
\hline & & & Trial 7 & 18.75 \\
\hline & & & Trial 6 & 19.23 \\
\hline \multirow{4}{*}{$\tau_{5}$} & \multirow{4}{*}{82} & \multirow{4}{*}{4} & Trial 1 & 10.91 \\
\hline & & & Trial 2 & 40.05 \\
\hline & & & Trial 4 & 19.32 \\
\hline & & & Trial 10 & 47.09 \\
\hline$\tau_{6}$ & 25 & 1 & Trial 8 & 7.51 \\
\hline$\tau_{7}$ & 27 & 0 & - & - \\
\hline \multirow{2}{*}{$\tau_{8}$} & \multirow[t]{2}{*}{55} & \multirow[t]{2}{*}{2} & Trial 1 & 39.41 \\
\hline & & & Trial 8 & 38.02 \\
\hline \multirow{3}{*}{$\tau_{9}$} & \multirow{3}{*}{46} & \multirow{3}{*}{3} & Trial 2 & 28.91 \\
\hline & & & Trial 3 & 29.78 \\
\hline & & & Trial 9 & 20.03 \\
\hline \multirow{4}{*}{$\tau_{10}$} & \multirow{4}{*}{100} & \multirow{4}{*}{4} & Trial 1 & 12.01 \\
\hline & & & Trial 3 & 27.09 \\
\hline & & & Trial 8 & 81.53 \\
\hline & & & Trial 9 & 82.09 \\
\hline \multirow{3}{*}{$\tau_{11}$} & \multirow{3}{*}{68} & \multirow{3}{*}{3} & Trial 5 & 51.79 \\
\hline & & & Trial 9 & 28.06 \\
\hline & & & Trial 10 & 31.55 \\
\hline \multirow{2}{*}{$\tau_{12}$} & \multirow[t]{2}{*}{39} & \multirow[t]{2}{*}{2} & Trial 1 & 11.77 \\
\hline & & & Trial 7 & 12.33 \\
\hline \multirow{3}{*}{$\tau_{13}$} & \multirow{3}{*}{48} & \multirow{3}{*}{3} & Trial 5 & 39.60 \\
\hline & & & Trial 6 & 40.79 \\
\hline & & & Trial 10 & 26.56 \\
\hline
\end{tabular}

\section{ACKNOWLEDGMENTS}

We also thank reviewers for their thorough advices in improving this article.

\section{REFERENCES}

A. Ajoudani, M. Gabiccini, N. G. Tsagarakis, and A. Bicchi. 2013. Human-like impedance and minimum effort control for natural and efficient manipulation. In Proceedings of the IEEE International Conference on Robotics and Automation. 4499-4505.

J. F. Allen. 1983. Maintaining knowledge about temporal intervals. Communications of the ACM 26, 11 (1983), 832-843.

P. Althaus and H. I. Christensen. 2003. Smooth task switching through behaviour competition. Robotics and Autonomous Systems 44, 3-4 (2003), 241-249.

K. Apt and M. Wallace. 2006. Constraint Logic Programming using Eclipse. Cambridge University Press, New York.

A. R. Aron. 2007. The neural basis of inhibition in cognitive control. The Neuroscientist 13 (2007), 214-228.

T. Baar, B. Beckert, and P. H. Schmitt. 2001. An extension of dynamic logic for modelling ocls @pre operator. In Perspectives of System Informatics, D. Bjrner, M. Broy, and A.V. Zamulin (Eds.). Lecture Notes in Computer Science, Vol. 2244. Springer, Berlin, 47-54. 
M. Beetz, L. Mosenlechner, and M. Tenorth. 2010. CRAM: A cognitive robot abstract machine for everyday manipulation in human environments. In Proceedings of the IEEE/RSJ International Conference on Intelligent Robots and Systems. 1012-1017.

S. A. Block, A. F. Wehowsky, and B. C. Williams. 2006. Robust execution on contingent, temporally flexible plans. In Proceedings of the AAAI Workshop on Cognitive Robotics. AAAI Press, Palo Alto, California, 802-808.

A. Blum and J. C. Langford. 1999. Probabilistic planning in the graphplan framework. In Proceedings of the 5th European Conference on Planning. Springer-Verlag, New York, NY, 8-12.

A. M. Buchanan and A. W. Fitzgibbon. 2005. Damped newton algorithms for matrix factorization with missing data. In Proceedings of the IEEE Conference on Computer Vision and Pattern Recognition. 316-322.

B. Cafaro, M. Gianni, F. Pirri, M. Ruiz, and A. Sinha. 2013. Terrain traversability in rescue environments. In Proceedings of the IEEE International Symposium on Safety, Security, and Rescue Robotics. 1-8.

J. F. Canny. 2002. Collaborative filtering with privacy via factor analysis. In Proceedings of the 25th Annual International ACM SIGIR Conference on Research and Development in Information Retrieval. 238-245.

D. Cao, Q. Qu, C. Li, and C. He. 2009. Research of attitude estimation of UAV based on information fusion of complementary filter. In Proceedings of the 4th International Conference on Computer Sciences and Convergence Information Technology. IEEE, 1290-1293.

G. Capi. 2007. Robot task switching in complex environments. In Proceedings of the IEEE/ASME International Conference on Advanced Intelligent Mechatronics. IEEE, 1-6.

G. Capi, G. G. Pojani, and S. I. Kaneko. 2008. Evolution of task switching behaviors in real mobile robots. In Proceedings of the 3rd International Conference on Innovative Computing Information and Control. IEEE, 495-501.

A. Carbone, A. Finzi, A. Orlandini, and F. Pirri. 2008. Model-based control architecture for attentive robots in rescue scenarios. Autonomous Robots 24, 1 (2008), 87-120.

P. Chen. 2008. Optimization algorithms on subspaces: Revisiting missing data problem in low-rank matrix. International Journal of Computer Vision 80, 1 (2008), 125-142.

M. M. Cialdea and F. Pirri. 1993. First order abduction via tableau and sequent calculi. Logic Journal of the IGPL 1, 1 (1993), 99-117.

D. B. D'Ambrosio, J. Lehman, S. S. Risi, and K. O. Stanley. 2011. Task switching in multirobot learning through indirect encoding. In Proceedings of the IEEE/RSJ International Conference on Intelligent Robots and Systems. IEEE, 2802-2809.

R. Dechter, I. Meiri, and J. Pearl. 1991. Temporal constraint networks. Artificial Intelligence 49, 1-3 (1991), 61-95.

X. Ding and C. Fang. 2013. A novel method of motion planning for an anthropomorphic arm based on movement primitives. IEEE/ASME Transactions on Mechatronics 18, 2 (April 2013), 624-636.

K. T. Durkee, C. Shabarekh, C. Jackson, and G. Ganberg. 2011. Flexible autonomous support to aid context and task switching. In Proceedings of the IEEE First International Multi-Disciplinary Conference on Cognitive Methods in Situation Awareness and Decision Support. IEEE, 204-207.

R. Fikes and N. J. Nilsson. 1971. STRIPS: A new approach to the application of theorem proving to problem solving. Artificial Intelligence 2, 3/4 (1971), 189-208.

M. Finger and D. Gabbay. 1996. Combining temporal logic systems. Notre Dame Journal of Formal Logic 37, 2 (1996), 204-232.

A. Finzi and F. Pirri. 2005. Representing flexible temporal behaviours in the situation calculus. In Proceedings of the 19th International Joint Conference on Artificial Intelligence. AAAI Press, 436-441.

A. Finzi and F. Pirri. 2010. Switching Tasks and Flexible Reasoning in the Situation Calculus. Technical Report 7. DIAG.

M. Fox and D. Long. 2003. PDDL2.1: An extension to PDDL for expressing temporal planning domains. Journal of Artificial Intelligence Research 20, 1 (2003), 61-124.

G. Ganesh and E. Burdet. 2013. Motor planning explains human behaviour in tasks with multiple solutions. Robotics and Autonomous Systems 61 (2013), 362-368. Issue 4.

M. Gianni, F. Ferri, and F. Pirri. 2013a. ARE: Augmented reality environment for mobile robots. In Proceedings of the 14th Towards Autonomous Systems (TAROS). 470-483.

M. Gianni, G. Gonnelli, A. Sinha, M. Menna, and F. Pirri. 2013b. An augmented reality approach for trajectory planning and control of tracked vehicles in rescue environments. In Proceedings of the IEEE International Symposium on Safety, Security, and Rescue Robotics. 1-6.

J. J. Gibson. 1960. The concept of the stimulus in psychology. American Psychologist 15, 11 (1960), 694-703.

M. Göbelbecker, C. Gretton, and R. Dearden. 2011. A switching planner for combined task and observation planning. In Proceedings of the 25th AAAI Conference on Artificial Intelligence. 
N. Hawes, M. Hanheide, K. Sjöö, A. Aydemir, P. Jensfelt, M. Göbelbecker, M. Brenner, H. Zender, P. Lison, I. Kruijff-Korbayová, G. J. M Kruijff, and M. Zillich. 2010. Dora the explorer: A motivated robot. In Proceedings of 9th International Conference on Autonomous Agents and Multiagent Systems, Vol. 1. International Foundation for Autonomous Agents and Multiagent Systems, Canada, 1617-1618.

D. Hurych, K. Zimmermann, and T. Svoboda. 2011. Fast learnable object tracking and detection in highresolution omnidirectional images. In Proceedings of the 9 th International Joint Conference on Computer Vision, Imaging and Computer Graphics Theory and Applications. SCITEPRESS, 521-530.

M. Ito, K. Noda, Y. Hoshino, and J. Tani. 2006. Dynamic and interactive generation of object handling behaviors by a small humanoid robot using a dynamic neural network model. Neural Networks 19 (2006), 323-337.

L. Jamone, B. Damas, J. Santos-Victor, and A. Takanishi. 2013. Online learning of humanoid robot kinematics under switching tools contexts. In Proceedings of the IEEE International Conference on Robotics and Automation. 4811-4817.

A. T. Jersild. 1927. Mental set and shift. Archives of Psychology 14, 89 (1927), 5-82.

S. Ko and J. Lee. 2002. User preference mining through collaborative filtering and content based filtering in recommender system. In Proceedings of the 3rd International Conference on E-Commerce and Web Technologies. 244-253.

V. Kubelka and M. Reinstein. 2012. Complementary filtering approach to orientation estimation using inertial sensors only. In Proceedings of the IEEE International Conference on Robotics and Automation. 599-605.

J. L. L. Herlocker, J. A. Konstan, L. G. Terveen, and J. T. T. Riedl. 2004. Evaluating collaborative filtering recommender systems. ACM Transactions on Information Systems 22, 1 (2004), 5-53.

Tao Li, K. Nakajima, and R. Pfeifer. 2013. Online learning for behavior switching in a soft robotic arm. In Proceedings of the IEEE International Conference on Robotics and Automation. 1296-1302.

X. Li and C. C. Cheah. 2012. Multiple task-space robot control: Sense locally, act globally. In Proceedings of the IEEE International Conference on Robotics and Automation. 265-270.

Pierre Marquis. 1991. Extending abduction from propositional to first-order logic. In Fundamentals of Artificial Intelligence Research. 141-155.

D. V. McDermott. 2003. PDDL2.1 - The art of the possible? Commentary on Fox and Long. Journal of Artificial Intelligence Research 20 (2003), 145-148.

A. I. Medina Ayala, S. B. Andersson, and C. Belta. 2012. Probabilistic control from time-bounded temporal logic specifications in dynamic environments. In Proceedings of the IEEE International Conference on Robotics and Automation. 4705-4710.

E. K. Miller and J. D. Cohen. 2007. An integrative theory of prefrontal cortex function. Annual Review of Neuroscience 24 (2007), 167-202.

J. Milln, F. Renkens, J. Mourino, and W. Gerstner. 2004. Brain-actuated interaction. Artificial Intelligence 159 (2004), 241-259.

S. Monsell. 2003. Task switching. Trends in Cognitive Sciences 7, 3 (2003), 134-140.

P. H. Morris, N. Muscettola, and T. Vidal. 2001. Dynamic control of plans with temporal uncertainty. In Proceedings of the 17th International Joint Conference on Artificial Intelligence. 494-502.

M. Nakano, Y. Hasegawa, K. Funakoshi, J. Takeuchi, T. Torii, K. Nakadai, N. Kanda, K. Komatani, H. G Okuno, and H. Tsujino. 2011. A multi-expert model for dialogue and behavior control of conversational robots and agents. Knowledge-Based Systems Journal 24 (2011), 248-256. Issue 2.

A. Newell. 1990. Unified Theories of Cognition. Harvard University Press.

D. A. Norman and T. Shallice. 1986. Consciousness and Self-Regulation: Advances in Research and Theory, Vol. 4. Attention to action: Willed and automatic control of behaviour. Plenum Press.

P. Papadakis and F. Pirri. 2012. 3D mobility learning and regression of articulated, tracked robotic vehicles by physics-based optimization. In Proceedings of the 9th Workshop on Virtual Reality Interactions and Physical Simulations. 147-156.

F. Pirri. 2011. The well-designed logical robot: Learning and experience from observations to the Situation Calculus. Artificial Intelligence 175, 1 (2011), 378-415.

F. Pirri and R. Reiter. 1999. Some contributions to the metatheory of the situation calculus. Journal of the ACM 46, 3 (1999), 325-361.

F. Pirri and R. Reiter. 2000. Planning with natural actions in the situation calculus. In Logic-Based Artificial Intelligence. Kluwer Press, 213-231.

F. Pomerleau, F. Colas, S. Siegwart, and S. Magnenat. 2013. Comparing ICP variants on real-world data sets. Autonomous Robots 34, 3 (April 2013), 133-148. 
M. Quigley, K. Conley, B. Gerkey, J. Faust, T. Foote, J. Leibs, R. Wheeler, and A. Ng. 2009. ROS: An open-source Robot Operating System. In Proceedings of the Open-Source Workshop of the International Conference on Robotics and Automation.

D. Randell, Z. Cui, and A. Cohn. 1992. A spatial logic based on regions and connection. In Proceedings of the 3rd International Conference on Principles of Knowledge Representation and Reasoning. 165-176.

J. Rubinstein, D. E. Meyer, and J. E. Evans. 2001. Executive control of cognitive processes in task switching. Journal of Experimental Psychology: Human Perception and Performance 27, 4 (2001), 763-797.

C. O. Saglam and K. Byl. 2013. Stability and gait transition of the five-link biped on stochastically rough terrain using a discrete set of sliding mode controllers. In Proceedings of the IEEE International Conference on Robotics and Automation. 5675-5682.

T. Stoyanov, M. Magnusson, H. Andreasson, and A. J. Lilienthal. 2010. Path planning in 3D environments using the Normal Distributions Transform. In Proceedings of the IEEE/RSJ International Conference on Intelligent Robots and Systems. 3263-3268.

C. Sung, N. Ayanian, and D. Rus. 2013. Improving the performance of multi-robot systems by task switching. In Proceedings of the IEEE International Conference on Robotics and Automation. 2999-3006.

S. Suzuki, T. Sasaki, and F. Harashima. 2009. Visible classification of task-switching strategies in vehicle operation. In Proceedings of the 18th IEEE International Symposium on Robot and Human Interactive Communication. 1161-1166.

T. Takayuki Okatani and K. Deguchi. 2006. On the Wiberg algorithm for matrix factorization in the presence of missing components. International Journal of Computer Vision 72, 3 (2006), 329-337.

K. Talamadupula, J. Benton, S. Kambhampati, P. Schermerhorn, and M. Scheutz. 2010. Planning for humanrobot teaming in open worlds. ACM Transactions on Intelligent Systems and Technology 1, 2 (2010), 14:1-14:24.

L. Tao, K. Nakajima, M. Cianchetti, C. Laschi, and R. Pfeifer. 2012. Behavior switching using reservoir computing for a soft robotic arm. In Proceedings of the IEEE International Conference on Robotics and Automation. 4918-4924.

S. P. Tipper. 2001. Does negative priming reflect inhibitory mechanisms? A review and integration of conflicting views. The Quarterly Journal of Experimental Psychology 54 (2001), 321-343.

M. Vilain, H. Kautz, and P. Beek. 1986. Constraint propagation algorithms for temporal reasoning. In Readings in Qualitative Reasoning about Physical Systems. Morgan Kaufmann, 377-382.

J. Wawerla and R. T. Vaughan. 2009. Robot task switching under diminishing returns. In Proceedings of the IEEE/RSJ International Conference on Intelligent Robots and Systems. 5033-5038.

E. H. Weber. 1834. De pulsu, resorptione, audita et tactu. Annotationes anatomicae et physiologicae. Koehler, New York, NY.

J. Wittocx, M. Denecker, and M. Bruynooghe. 2010. Constraint propagation for extended first-order logic. Computing Research Repository abs/1008.2121 (2010).

F. Wolter and M. Zakharyaschev. 2000. Spatio-temporal representation and reasoning based on RCC-8. In Proceedings of the 7th International Conference on Principles of Knowledge Representation and Reasoning. 3-14.

J. L. Wyatt, A. Aydemir, M. Brenner, M. Hanheide, N. Hawes, P. Jensfelt, M. Kristan, G. J. M. Kruijff, P. Lison, A. Pronobis, K. Sjöö, A. Vrecko, H. Zender, M. Zillich, and D. Skocaj. 2010. Self-understanding and self-extension: A systems and representational approach. IEEE Transactions on Autonomous Mental Development 2, 4 (2010), 282-303.

Z. Zhang, K. Zhao, and H. Zha. 2012. Inducible regularization for low-rank matrix factorizations for collaborative filtering. Neurocomputing 97 (2012), 52-62.

Y. Zhou, D. M. Wilkinson, R. Schreiber, and R. Pan. 2008. Large-scale parallel collaborative filtering for the netflix prize. In Proceedings of the 4th International Conference on Algorithmic Aspects in Information and Management. 337-348.

Received February 2013; revised August 2014; accepted October 2014 